\title{
Microsphere embolism-induced cortical cholinergic deafferentation and impairments in attentional performance
}

\author{
Tara K. S. Craft, ${ }^{1}$ John H. Mahoney, ${ }^{2}$ A. Courtney DeVries ${ }^{1}$ and Martin Sarter ${ }^{3}$ \\ ${ }^{1}$ Departments of Psychology and Neuroscience, Ohio State University, Ohio, USA \\ ${ }^{2}$ Department of Psychology, University of Colorado, Colorado, USA \\ ${ }^{3}$ Department of Psychology, University of Michigan, 525 E. University Ave, 4032 East Hall, Ann Arbor, MI 48109, USA
}

Keywords: acetylcholine, attention, cortex, ischemia, microsphere embolism, stroke

\begin{abstract}
Ischemic events have been hypothesized to play a critical role on the pathogenesis of dementia and the acceleration of cognitive impairments. This experiment was designed to determine the consequences of microvascular ischemia on the cortical cholinergic input system and associated attention capacities. Injections of microspheres $(\approx 50 \mu \mathrm{m}$ diameter; $\approx 5000$ microspheres $/ 100 \mu \mathrm{L})$ into the right common carotid artery of rats served as a model of microvascular ischemia and resulted in decreases in the density of cholinergic fibers in the ipsilateral medial prefrontal cortex and frontoparietal areas. Furthermore, dense astrogliosis, indicated by glial fibrillary acidic protein (GFAP) immunohistochemistry, was observed in the globus pallidus, including the areas of origin of cholinergic projections to the cortex. Fluoro-Jade B staining indicated that loss of neurons in the cortex was restricted to areas of microsphereinduced infarcts. Attentional performance was assessed using an operant sustained attention task; performance in this task was previously demonstrated to reflect the integrity and activity of the cortical cholinergic input system. Embolized animals' performance was characterized by a decrease in the animals' ability to detect signals. Their performance in non-signal trials remained unaffected. The residual density of cholinergic axons in prefrontal and frontoparietal areas correlated with the animals' performance. The present data support the hypothesis that microvascular ischemia results in loss of cortical cholinergic inputs and impairs associated attentional performance. Microsphere embolism represents a useful animal model for studying the role of interactions between microvascular disorder and impaired forebrain cholinergic neurotransmission in the manifestation of cognitive impairments.
\end{abstract}

\section{Introduction}

'Ministrokes' or, generally, microvascular disorder, represent significant and possibly necessary components of the neuropathological mechanisms resulting in Alzheimer's disease (de la Torre \& Stefano, 2000; Kalaria, 2002; Meyer et al., 2002; Seshadri et al., 2002; Zlokovic, 2002; Zekry et al., 2003; Langa et al., 2004). Vascular lesions in cortical associational regions predict the severity of dementia (Montaldi et al., 1990; Zekry et al., 2003), and Alzheimer's disease has even been conceptualized as a vascular disorder with neurodegenerative consequences (de la Torre \& Stefano, 2000).

Interactions between vascular and degenerative mechanisms may be essential for the manifestation of the cognitive symptoms of pathological ageing (Farkas \& Luiten, 2001; Sarter \& Bruno, 2004). The role of forebrain cholinergic systems in age-related neuropathological processes has been extensively studied (Terry \& Buccafusco, 2003; Mesulam, 2004). An impaired microvascular system may represent a major variable contributing to the vulnerability of these neurons. Basal forebrain cholinergic projections innervate and dilate cortical microvessels directly, as well as indirectly via a local cortical nitric oxide relay (Raszkiewicz et al., 1992; Vaucher \& Hamel, 1995; Luiten et al., 1996; Vaucher et al., 1997a; Barbelivien et al., 1999). Ischemic events and

Correspondence: Martin Sarter, as above.

E-mail: msarter@umich.edu

Received 7 March 2005, revised 5 April 2005, accepted 6 April 2005 microvascular disorder have been suggested to reduce cortical cholinergic activity and cholinergic innervation (Kalaria, 2002). Conversely, decreased cholinergic activity impairs cortical perfusion and metabolic activity and thus enhances the risk for ischemic events (Zhang et al., 1997; Tong \& Hamel, 1999; Harkany et al., 2000; Farkas \& Luiten, 2001; Zlokovic, 2002). These interactions are of particular importance with respect to ageing and dementia, because abnormalities in the regulation of cholinergic neurons have been documented early in the disease process (Mufson et al., 2000, 2002; Chu et al., 2001; Mesulam, 2004), and loss of cortical cholinergic inputs correlates with the severity of the cognitive symptoms of Alzheimer's disease (Perry et al., 1981; Sims et al., 1983; Lehericy et al., 1993; Palmer, 1996; Procter, 1996; Geula, 1998; Pappas et al., 2000).

The present experiment was designed to test the hypothesis that microvascular disorder affects the integrity of the cortical cholinergic input system and impairs the performance of a task known to reflect the status of the cortical cholinergic input system (Arnold et al., 2002; Sarter et al., 2005). In order to model cerebral microcirculatory insufficiency (de la Torre \& Stefano, 2000; Reed, 2004), microspheres $(50 \mu \mathrm{m}$ diameter) were injected into the common carotid artery of rats. Microspheres were expected to move primarily through middle and anterior cerebral arteries and lodge in arterioles and capillaries, thereby attenuating the perfusion of frontal and parietal regions (Kataoka et al., 1991; Takeo et al., 1992; Miyake et al., 1993; Takagi et al., 1997b).

Microsphere embolism has been previously demonstrated to affect forebrain cholinergic (Kataoka et al., 1991; Takagi et al., 1997a; 
Takagi et al., 1997b) and other neurotransmitter systems (Takagi et al., 1995; Fukatsu et al., 2002), and to impair behavioural and cognitive functions measured by using standard behavioural tests (references above). The present experiment was intended to quantify the loss of cortical cholinergic inputs and to investigate the relationships between this loss and the performance of the animals in a task assessing attentional functions. Performance in this task has been demonstrated to depend on the integrity of the cortical cholinergic input system (McGaughy et al., 1996; McGaughy \& Sarter, 1998) and to be associated with increases in cortical acetylcholine (ACh) release (Arnold et al., 2002). Additional analyses utilized glial fibrillary acidic protein (GFAP) immunohistochemistry and the Fluoro-Jade method (Schmued \& Hopkins, 2000) in order to generate insights into the mechanisms that may underlie the effects of microsphere embolism on the cholinergic system. Collectively, these experiments were expected to illustrate the usefulness of this model in research on the role of interactions between microvascular disorder and cholinergic dysregulation in the development of cognitive impairments.

\section{Materials and methods}

\section{Subjects}

Subjects were male BNNIA/F344 rats (Harlan Sprague-Dawley, Indianapolis, IN), aged three months at the beginning of the experiments. Rats were housed individually in a temperature and humidity controlled vivarium with a 12-h light : 12-h dark schedule (lights on $02: 00 \mathrm{~h}$, off 14:00 h). Rats used for the behavioural experiments were moderately water-deprived (maintained at $85 \%$ of their pre-experimental weight), by providing water as reward in daily test sessions (below) and ad libitum for $8 \mathrm{~min}$ after each session, and with food always available ad libitum. All experiments were conducted in accordance with the 'US Government Principles for the Utilization and Care of Vertebrate Animals used in Testing, Research, and Training' in an AAALAC-accredited facility and based on protocols approved by the University Institutional Laboratory Care and Use Committee.

\section{Microsphere injections, justification for right carotid injections, and experimental groups}

Under general anaesthesia (1-3\% isoflurane gas delivered by nose cone, Anesco/Surgivet, Waukesha, WI), rats were placed on a Deltaphase isothermal pad (Braintree Scientific, Inc., Braintree, Mass.) and temperature was maintained at $37{ }^{\circ} \mathrm{C}$. In a supine position, a midline neck incision was made to identify the common carotid artery and the bifurcation to the internal and external carotid arteries. The common carotid artery and external carotid arteries were separately ligated with suture. Saline or microspheres (composed of styrenedivinyl benzene; $50 \mu \mathrm{m}$ in diameter; suspended in $10 \%$ dextrane and $0.01 \%$ Tween; PerkinElmer Instruments; Shelton, CT; $\approx 5000$ spheres $/ 100 \mu \mathrm{L} ; 100 \mu \mathrm{L} / \mathrm{min}$ ) were injected via a $30-\mathrm{G}$ needle into the right common carotid artery, superior to the ligation site but inferior to the bifurcation of the internal and external carotid arteries. The needle remained in the artery for one minute postinjection and the artery was observed for bleeding after withdrawal before the ligating sutures were removed and normal blood-flow was restored.

The right hemisphere was selected for microsphere embolism because the integrity of right, but not left, cholinergic inputs to the cortex is required for normal attentional performance (Martinez \& Sarter, 2004). Therefore, left hemispheric embolism was not expected to yield robust impairments in attentional performance and incon- clusive relationships between histological and behavioural measures (see also Apparsundaram et al., 2005).

The main experimental groups and analyses are summarized in Table 1. Because of the focus on the effects of microvascular disorder on the integrity of forebrain cholinergic systems, initial experiments assessed the effects of two doses of microspheres on the density of cholinergic fibers in telencephalic regions. Based on these experiments, all subsequent experiments were based on injections of $250 \mu \mathrm{L}$. In order to obtain maximal Fluoro-Jade stain and GFAPpositive labelling (see below for details), a separate cohort of animals was processed for histological analyses 1 week after injections of microspheres or saline (Hopkins et al., 2000; Haga et al., 2002). Laser Dopper flowmetry (LDF) was used to measure cerebral blood flow following microsphere injection in a separate group of animals. Finally, in attention task performing animals, the effects of microsphere embolism on performance was assessed and, 16 weeks postinjection, these brains were processed for AChE histochemistry, and residual fibre densities were correlated with performance and compared with AChE-positive fibre densities observed 2 weeks postinjection (see Table 1).

\section{AChE histochemistry and quantification of AChE-positive fibre density}

Rats were anaesthetized with sodium pentobarbital $(150 \mathrm{mg} / \mathrm{kg}$ i.p.) and transcardially perfused with heparinized saline and $10 \%$ formalin followed by removal of the brain. These brains remained overnight in formalin prior to being dehydrated in a 30\% sucrose solution for cryoprotection. Brains were sectioned at $40 \mu \mathrm{m}$ in a cryostat (Leica Microsystems, Inc., Deerfield, IL) and stained using a modified procedure that optimizes the visualization of acetylcholinesterase (AChE)-positive axons (Tago et al., 1986). Briefly, sections were rinsed in $0.1 \% \mathrm{H}_{2} \mathrm{O}_{2}$ for $10 \mathrm{~min}$ prior to being rinsed with $0.1 \mathrm{M}$ maleate buffer ( $\mathrm{pH}$ 6.0). After rinsing, sections were incubated in a solution of $5 \mathrm{mg}$ acetylcholine iodide, $0.447 \mathrm{~g}$ sodium citrate, $0.025 \mathrm{~g}$ potassium ferricyanide, and $0.112 \mathrm{~g}$ cupric sulphate in $200 \mathrm{~mL}$ maleate buffer ( $\mathrm{pH}$ 6.0). After rinsing with $50.0 \mathrm{mM}$ Tris buffer (pH 7.6), staining was visualized using a solution of $0.050 \mathrm{~g}$ diaminobenzidine (DAB) and enhanced with $0.375 \mathrm{~g}$ nickel ammonium sulphate in $125.0 \mathrm{~mL}$ of $50 \mathrm{~mm}$ Tris buffer $(\mathrm{pH} \mathrm{6.2)}$ ). After $10 \mathrm{~min}$, eight drops of $0.1 \% \mathrm{H}_{2} \mathrm{O}_{2}$ were added. Sections continued to incubate for approximately $15 \mathrm{~min}$.

TABLE 1. Main experimental groups and analyses

\begin{tabular}{lcc}
\hline Analyses and injections & Number of rats & Volume injected $(\mu \mathrm{L})$ \\
\hline AChE histochemistry; Nissl stain (2-week survival) & \\
Microspheres & 6 & 100 \\
Vehicle & 6 & 100 \\
Microspheres & 6 & 250 \\
Vehicle & 6 & 250 \\
GFAP immunohistochemistry; Fluoro-Jade B staining & $(1$-week survival) \\
Microspheres & 7 & 250 \\
Vehicle & 5 & 250 \\
Laser Doppler flowmetry (3-15 min postinjection) & \\
Microspheres & 7 & 250 \\
Vehicle & 5 & 250 \\
Attentional task performance (16 weeks); AChE histochemistry; Nissl stain \\
Microspheres & 8 & 250 \\
Vehicle & 8 & 250 \\
&
\end{tabular}


A modified grid-counting method was used to generate semiquantitative estimates of the density of cortical AChE-positive axons (Stichel \& Singer, 1987; see also Fadel et al., 1996; Turchi \& Sarter, 2000; Burk et al., 2002). For most cortical areas, the distribution of AChE-positive fibers closely matches that of choline acetyltransferase (ChAT)-fibers (Lysakowski et al., 1989). Briefly, at a magnification of $40 \times$, the focusing magnifier of a VANOX Olympus Research Microscope (Olympus America, Melville, NY) was inserted such that four orthogonal double cross lines were superimposed over the cortical region of interest. All fibers crossing the double lines were counted. For the cortical areas selected for fibre counting [medial prefrontal, frontoparietal (separate counts were taken for layers V/VI and I-IV)], three counts were taken per hemisphere from three different sections and averaged. Parallel sections were stained using cresyl violet to identify the location and extent of microsphere-induced infarcts. As most microspheres were washed out by these procedures, several 100$\mu \mathrm{m}$-thick sections were taken from each brain and individually thawmounted and Nissl-stained by applying all washes and dyes via syringe across the surface of the slide in order to document microspheres lodged in the brain.

\section{GFAP immunohistochemistry}

Activation of astrocytes was documented by immunohistochemical staining for GFAP. Rats were anaesthetized with sodium pentobarbital $(150 \mathrm{mg} / \mathrm{kg}$ i.p.) and transcardially perfused with heparinized saline ( $5 \mathrm{~min}$ ), followed by $500 \mathrm{~mL}$ of $4 \%$ paraformaldehyde. Brains were removed from the skull and dehydrated in a $30 \%$ sucrose solution for cryoprotection. Once dehydrated, brains were stored at $-70{ }^{\circ} \mathrm{C}$ until sectioning. Coronal sections were produced using a cryostat (Leica Microsystems, Inc., Deerfield, IL), alternating between $40-\mu \mathrm{m}$ sections free-floated in PBS (0.1 M; $\mathrm{pH} 7.2)$ for immunocytochemistry, and $25 \mu \mathrm{m}$ sections mounted on $2 \%$ gelatinized slides for histofluorescent labelling (below). Free-floating sections were first washed in PBS, then incubated in $10 \%$ normal goat serum (Chemicon, Temecula, CA) for $1 \mathrm{~h}$ on a shaker at room temperature, followed by $24 \mathrm{~h}$ incubation in primary antibody (1:40000 dilution of rabbit anti-GFAP (Dakocytomation, Carpinteria, $\mathrm{CA}$; in $0.3 \%$ Triton-X/PBS solution) at $4{ }^{\circ} \mathrm{C}$. Sections were then washed and exposed to biotinylated secondary antibody (Vector Laboratories, Burlingame, CA) at a $1: 200$ dilution in PBS for $1 \mathrm{~h}$. Sections were washed again, then incubated in avidin-biotin complex (ABC; Vector Laboratories), prepared according to Vector Laboratory instructions. After $1 \mathrm{~h}$ on a shaker, sections were washed, then exposed to a solution of DAB (Vector Laboratories), $\mathrm{NiCl}, \mathrm{H}_{2} \mathrm{O}_{2}$, and $\mathrm{PBS}$ for visualization. Following a final wash, sections were mounted on $2 \%$ gelatinized slides, dehydrated, and cover-slipped with Permount.

\section{Fluoro-Jade $B$ histofluorescence}

Fluoro-Jade B (FJ) is a fluorochrome that labels neurons undergoing degeneration (Schmued \& Hopkins, 2000). Mounted 25- $\mu$ m sections were dried at room temperature before proceeding to the standard Fluoro-Jade B ${ }^{\circledR} \quad$ (Histo-Chem, Jefferson, AR) staining protocol (Schmued \& Hopkins, 2000). Slides were immersed in 100\% alcohol for $3 \mathrm{~min}$, followed by $1 \mathrm{~min}$ in $70 \%$ alcohol and $1 \mathrm{~min}$ in distilled water. Slides were then transferred into a solution of $0.06 \%$ potassium permanganate, and gently agitated for $15 \mathrm{~min}$. Following a 1-min rinse in distilled water, the slides were immersed in a $0.001 \%$ staining solution (made from $0.01 \%$ Fluoro-Jade $\mathrm{B}^{\circledR}$ stock solution and $0.1 \%$ acetic acid vehicle) for $30 \mathrm{~min}$, with gentle agitation and light protection. Following staining, the slides were rinsed three times in distilled water for $1 \mathrm{~min}$, then allowed to air-dry in the dark. As a final step, the dried slides were immersed in xylene for clearing, then coverslipped with DPX (Sigma, St. Louis, MO). Sections were examined using a Leica HC fluorescence microscope equipped with a Leica I3 Filtercube (Leica Microsystems, Bannockburn, IL), a Sony 3CCD colour video camera (DXC-970 MD), and using MCID-Elite imaging analysis software (Imaging Res. Inc., St. Catharines, Ontario, Canada).

\section{Laser Doppler flowmetry}

Relative cerebral blood flow was determined in a separate cohort of animals (see Table 1). Rats were anaesthetized with 1-3\% isoflurane and secured to the nose cone to minimize movement. A 2-3-mm diameter closed cranial window was thinned with a hand-held drill in the right anterior parietal bone $(2 \mathrm{~mm}$ posterior and $6 \mathrm{~mm}$ lateral to the bregma) for placement of a Laser Doppler flowmetry (LDF) probe (Moor Instruments, Ltd, Model MBF3D, England), as previously described (Alkayed et al., 1998). The rat was then rotated along its longitudinal axis onto its dorsum, and the LDF probe secured in the cranial window. LDF readings were recorded after ligation of the common carotid artery and external carotid artery (baseline), immediately following the injection of saline or microspheres, and 3, 5, 10, and 15 min postinjection.

\section{Training and testing of sustained attention performance}

A computer-controlled operant system, consisting of 12 operant chambers (MedAssociates, East Fairfield, VT), was used. Each sound attenuated operant chamber was equipped with an overhead houselight $(2.8 \mathrm{~W})$ and a ventilation fan that also provided ambient background white noise. Operant boxes were equipped with three panel lights (2.8 W each; for luminance values see Holley et al., 1995) located above two retractable response levers. A water dispenser was located at the same level as the levers on the opposite wall of the intelligence panel. Medical-PC software (Version IV) was used with an IBM-PC clone to control input and output functions.

Details about the training of the animals in the sustained attention task, including evidence in support of the validity of performance measures in terms of indicating sustained attention performance, and the necessary role of the cortical cholinergic input system for sustained attention performance, were described previously (McGaughy \& Sarter, 1995, 1998; Turchi \& Sarter, 2001; Arnold et al., 2002). Behavioural training and testing was carried out daily between 08:00 $\mathrm{h}$ and 11:00 h EST, approximately 6-9 h after lights on. Briefly, animals were initially trained to bar press for water using an FR1 schedule of reinforcement with each lever press resulting in water delivery. Thereafter, animals were trained to lever press in accordance with the following rules. One second following a signal (illumination of the central panel light for $1 \mathrm{~s}$ ) or a non-signal event (no illumination), both levers were extended. Following a signal, a left lever press was rewarded ( $33 \mu \mathrm{L}$ water) and counted as a hit, while a right lever press indicated a miss and was not rewarded. If the levers were inserted after a non-signal event, a right lever press constituted a correct rejection and was rewarded, while a left lever press was counted as a false alarm. If no response occurred within $4 \mathrm{~s}$ following lever insertion, levers were withdrawn and an error of omission was counted. Signal and non-signal events occurred unpredictably with an intertrial interval of $9 \pm 3 \mathrm{~s}$ (McGaughy \& Sarter, 1995).

Because the potential contributions of contralateral visuospatial (Bushnell et al., 1998; Oshiro et al., 2000) or sensorimotor neglect, 
lateralized forelimb use (Schallert et al., 2000; Gharbawie \& Whishaw, 2003), or other lateralized functions (Soblosky et al., 1996; Ward et al., 1997), to the effects of microsphere embolism on attentional performance, behavioural training and testing were conducted in half of the animals using the reversed propositional rules for responding in signal and non-signal trials.

After animals generated $>65 \%$ correct lever presses to both signal and non-signal trials over a minimum of three consecutive training sessions, 1-s signals were replaced by signals lasting 500,50 , or $25 \mathrm{~ms}$. The final task consisted of 81 signal ( 27 per signal duration) and 81 non-signal trials per session. Trials were presented in a pseudorandom sequence to allow for a posthoc analysis of the performance data across three blocks of 54 trials each (27 signal trials, nine per signal duration; 27 non-signal trials). A daily session lasted approximately $40 \mathrm{~min}$. Animals required approximately 12 weeks of training to reach stable criterion performance, defined as $>65 \%$ hits to 500 -ms signals, $>65 \%$ correct rejections, and $<24$ errors of omissions. After reaching criterion, animals underwent surgery. Following a 7-day postsurgery recovery period, rats were re-trained in the sustained attention task for 14 days or until asymptotic performance was achieved (defined by $<10 \%$ variability in hits and correct rejections over three consecutive sessions). Thereafter, the effects of a visual distracter, consisting of the houselights flashing on-off at $0.5 \mathrm{~Hz}$ throughout the sessions (McGaughy \& Sarter, 1995), were tested in embolized and control animals.

\section{Statistical analyses}

The relative number of hits and correct rejections were normalized (Zar, 1974) and the transformed values $\left[X^{\prime}=2 \operatorname{arcsine} x^{1 / 2}\right]$ were analysed using mixed ANOVAs. Within-subject variables included (where appropriate) presurgery vs. postsurgery, block of trials, and signal duration, while embolism was a between-subject variable. Independent sample $t$-tests and paired sample $t$-tests were used to locate significant effects and interactions indicated by ANOVA. The effects of embolism on performance were analysed by comparing the average of the measures taken from the last five test sessions prior to surgery with the data from the last three sessions prior to the presentation of the distractor. The latter formed the baseline for the assessment of the effects of the distractor. Differences in relative cerebral blood flow during carotid injections were analysed using repeated measures ANOVA, followed by posthoc analysis using Fisher's PLSD Test. Finally, Pearson's correlation coefficients were calculated to explore the relationships between the animals' performance and residual AChE-fibre density in the cortex $(\alpha=0.05$ for all tests). Statistical analyses were performed using the SPSS/PC +11.0 Version (SPSS Int., Chicago, Il.).

\section{Results}

\section{Laser Doppler flowmetry}

There were no significant group differences in mean relative cerebral blood flow at any time point during the carotid injection procedure $\left[F_{1,10}=0.092, \quad P>0.05 ;\right.$ immediately following the injection (saline/micropshere), $98.2 \pm 1.11 \% / 102.0 \pm 2.51 \%$; $3 \mathrm{~min}$ postinjection, $\quad 93.6 \pm 2.87 \% / 93.4 \pm 5.34 \% ; \quad 5 \mathrm{~min}$ postinjection, $91.4 \pm 2.00 \% / 90.2 \pm 7.07 \% ; 10 \mathrm{~min}$ postinjection, $87.2 \pm 6.29 \%$ / $90.8 \pm 7.59 \% ; \quad 15 \mathrm{~min} \quad$ postinjection, $130.1 \pm 21.75 \%$ / $112.4 \pm 7.70 \%$ ]. Thus, as expected, injections of microspheres into the carotid did not result in significant changes in blood flow in the anterior parietal cortex.

\section{Infarcts and large-diameter blood vessels}

Microsphere-induced infarcts, defined by relatively large $(>100 \mu \mathrm{m}$ diameter) areas of gliosis, were not consistently observed. Furthermore, there was no apparent relationship between the occurrence of such infarcts, their location, and the decreases in cortical AChEpositive fibre density, which were observed consistently in embolized brains (below). Likewise, astrogliosis in cortical and subcortical areas, as indicated by GFAP-immunoreactivity (below), was not necessarily associated with the presence of large infarcts (see Fig. 1 for examples). Collectively, large infarcts and haemorrhages did not seem to represent reliable consequences of microsphere embolism, and they did not seem to be associated with the decreases in the density of cortical AChE-positive fibers or subcortical and cortical astrogliosis (below).

In embolized animals, a high density of large diameter blood vessels (Knox \& Oliveira, 1980) was observed particularly in fronto-medial and fronto-parietal cortical regions (Fig. 2). The diameter of these vessels consistently matched that of the microspheres $(50 \mu \mathrm{m})$, suggesting that microspheres passed through and/or were lodged preferentially in these regions. Furthermore, GFAP-positive, astrocytic proliferation typically surrounded these large-diameter vessels (Fig. 2).

\section{Cortical cholinergic deafferentation}

Injection of microspheres into the carotid artery resulted in a reduction of the density of cortical AChE-positive axons. The decrease was most pronounced in the medial prefrontal cortex and the outer layers of frontoparietal cortical areas (Fig. 3, Table 2).

As summarized in Table 1, injection of $100 \mu \mathrm{L}(250 \mu \mathrm{L})$ of microsphere suspension produced a $44 \%(52 \%)$ decrease in AChEpositive fibre density in the ipsilateral medial prefrontal cortex and a $34 \%(40 \%)$ decrease in the outer layers of the frontoparietal cortex when compared to sham-operated animals. Collectively, comparisons between the counts obtained from the hemisphere ipsilateral to the injection of microspheres or saline (between-group comparisons), and between the counts obtained from the ipsilateral and contralateral hemispheres (between-hemispheres comparisons; Table 2) indicate that the loss of AChE-positive fibers was largely restricted to the hemisphere ipsilateral to the infusion of microspheres into the carotid artery. Decreases in AChE-positive axons were less pronounced in the posterior half of the cortex and, as illustrated in Fig. 1, were not observed in the hippocampus. Collectively, microsphere embolism produced marked and dosedependent reductions in the density of cholinergic inputs to frontal cortical regions.

\section{Astrocyte activation (GFAP-immunohistochemistry)}

Dense astrogliosis in the globus pallidus (GP), ipsilateral to the microsphere injection, was a consistent result of microsphere embolism (Fig. 4). GFAP-positive cells were only sparsely distributed in the contralateral globus pallidus (Fig. 4), matching the occurrence of such cells in the ipsilateral globus pallidus of saline-injected rats.

In the cortex, astrogliosis occurred in restricted areas, forming 'pockets' as shown in Fig. 5A, or was more diffusely distributed in the area surrounding a lodged microsphere (Fig. 5B). Furthermore, activated astrocytes were observed in the corpus callosum adjacent to the prelimbic cortex (Fig. 5C), but not in the prelimbic cortex where the decreases in AChE-positive fibre density were observed (above). 

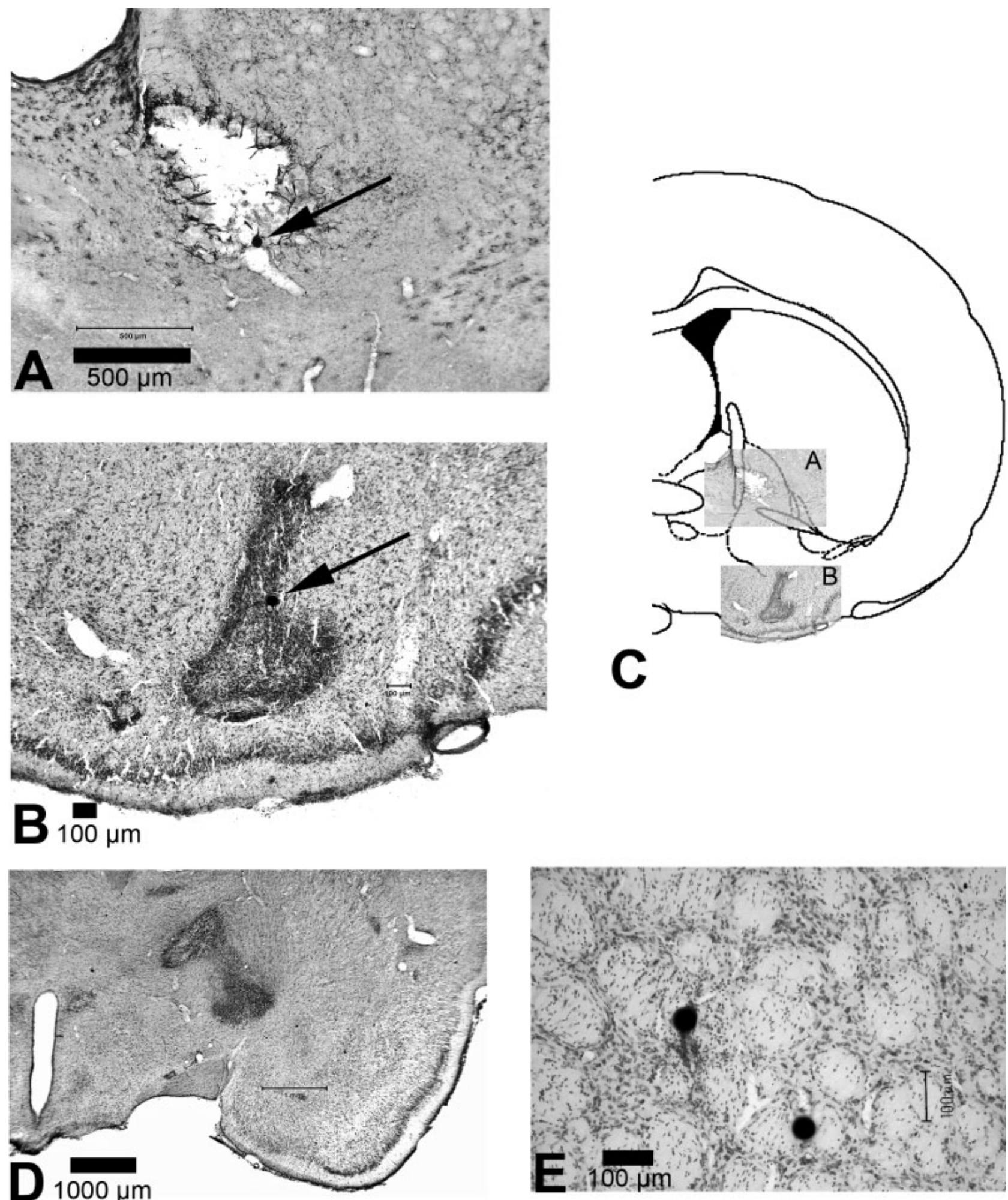

FIG. 1. Examples of the microsphere-induced infarcts. (A) GFAP-stained section showing the tissue loss that resulted from an infarct and subsequent tissue processing (the arrow points to a microsphere lodged in this section). This infarct affected the anterior portion of the medial globus pallidus and the internal capsula (see the location of this area superimposed on a schematic section in C). (B) Shows an infarct that resulted in the degeneration of parts of the horizontal nucleus of the diagonal band and preoptic nucleus (Nissl-stained section; the arrow points to a microsphere located in this section; the location of this area is also illustrated in C). It should be noted that microspheres were rarely observed in stained sections as they were washed out during sectioning and processing (B and E were thaw-mounted and carefully stained in order to capture microspheres; see Materials and methods). (D) Nissl-stained section showing the largest infarct observed across all brains; this infarct affected the posterior globus pallidus, internal capsula, and extended dorsomedially into the reticular thalamic nucleus. Such infarcts were inconsistently observed, they varied in location, and they did not seem to be associated systematically with the decreases in AChE-positive fibre density and cortical and subcortical astrogliosis, which were consistent consequences of microsphere embolism. (E) Shows the striatum of a thaw-mounted section; as was typically the case, relatively small areas of gliosis surrounded the location of a microsphere, and the occurrence of large infarcts remained the exception. 

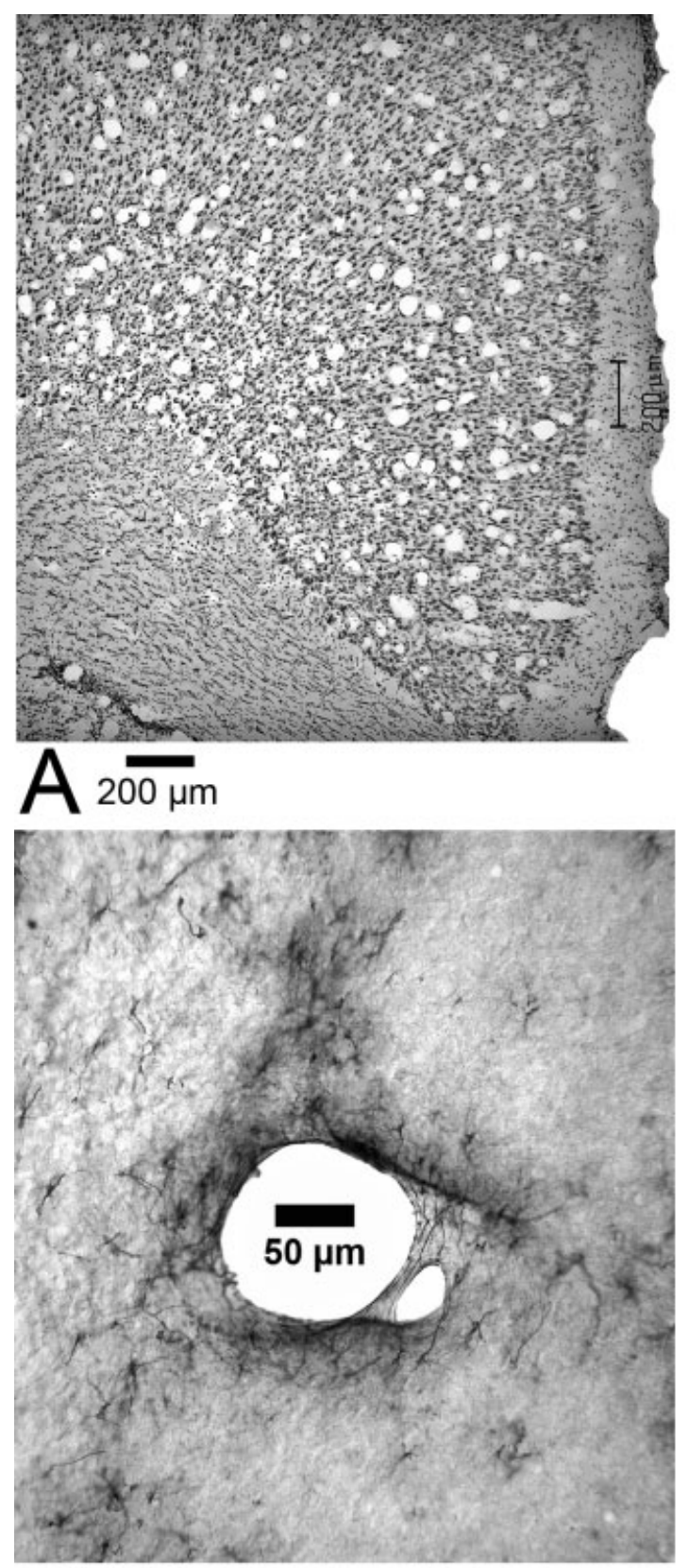

D
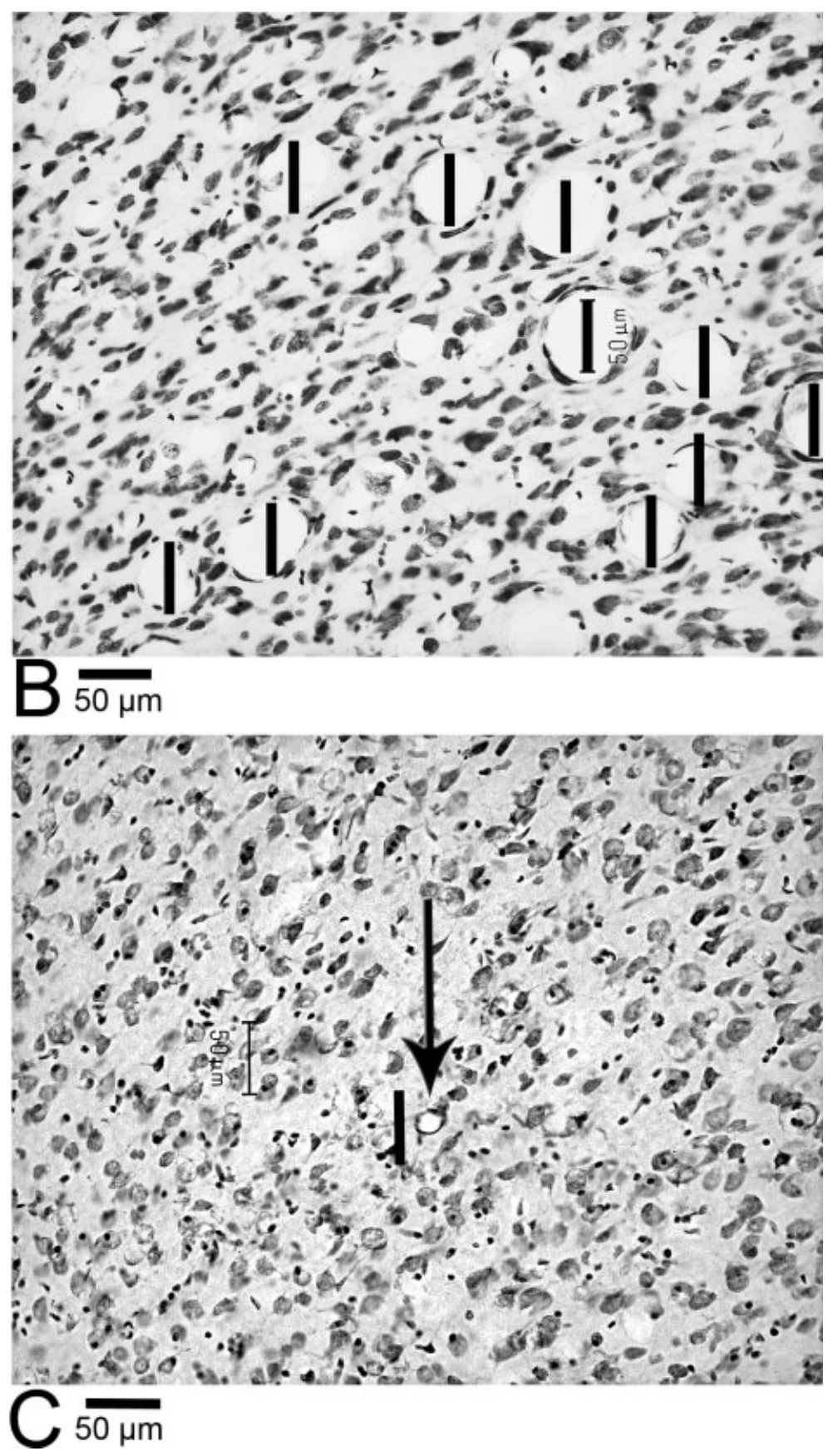

FIG. 2. (A) Photomicrograph from a Nissl-stained section of the anterior cingulate cortex of an embolized (100 $\mu \mathrm{L})$ rat showing a high density of enlarged cerebral vessels which, as shown at higher magnification in $\mathrm{B}$, are approximately $50 \mu \mathrm{m}$ in diameter (the microspheres used in this study were $50 \mu \mathrm{m}$ in diameter; in B, $50 \mu \mathrm{m}$ bars were inserted into the vessel space to illustrate this observation). In control rats (C), the diameters of vessels in this area varied between 25 and $40 \mu \mathrm{m}$ (see arrow and the adjacent $50 \mu \mathrm{m}$-calibration bar), and the density of such vessels was relatively low (see also Knox \& Oliveira, 1980; Pinard et al., 2002). (D) Microphotograph from a GFAP-stained section; astrocytic proliferation surrounding a large-diameter cortical blood vessel was consistently observed.

\section{Fluoro-Jade (FJ) histofluorescence}

Similar to the GFAP-positive pockets exemplified in Fig. 5A, pockets of FJ-labelled cells were found in the cortex, preferentially in frontoparietal regions (Fig. 6). Furthermore, bands of FJ-positive neurons were observed in layers $\mathrm{V}$ and VI of the prelimbic and anterior cingulate cortex. Outside the globus pallidus, subcortical FJlabelled neurons were only sparsely distributed, such as the labelled mediodorsal thalamic neurons shown in Fig. 6D.

\section{Microsphere embolism-induced impairments in attentional performance}

Pre- and postsurgery body weights of task-performing animals

Prior to surgery, the body weights of rats assigned to receive injections of microspheres or saline did not differ [Mann-Whitney $U=23.00$, $P>0.05 ;$ rats assigned to sham-surgery (Mean \pm SEM) $340.42 \pm 6.89 \mathrm{~g} ; \quad$ rats assigned to microsphere embolism, $338.71 \pm 3.03 \mathrm{~g}]$. Following the seven-day postsurgery recovery 

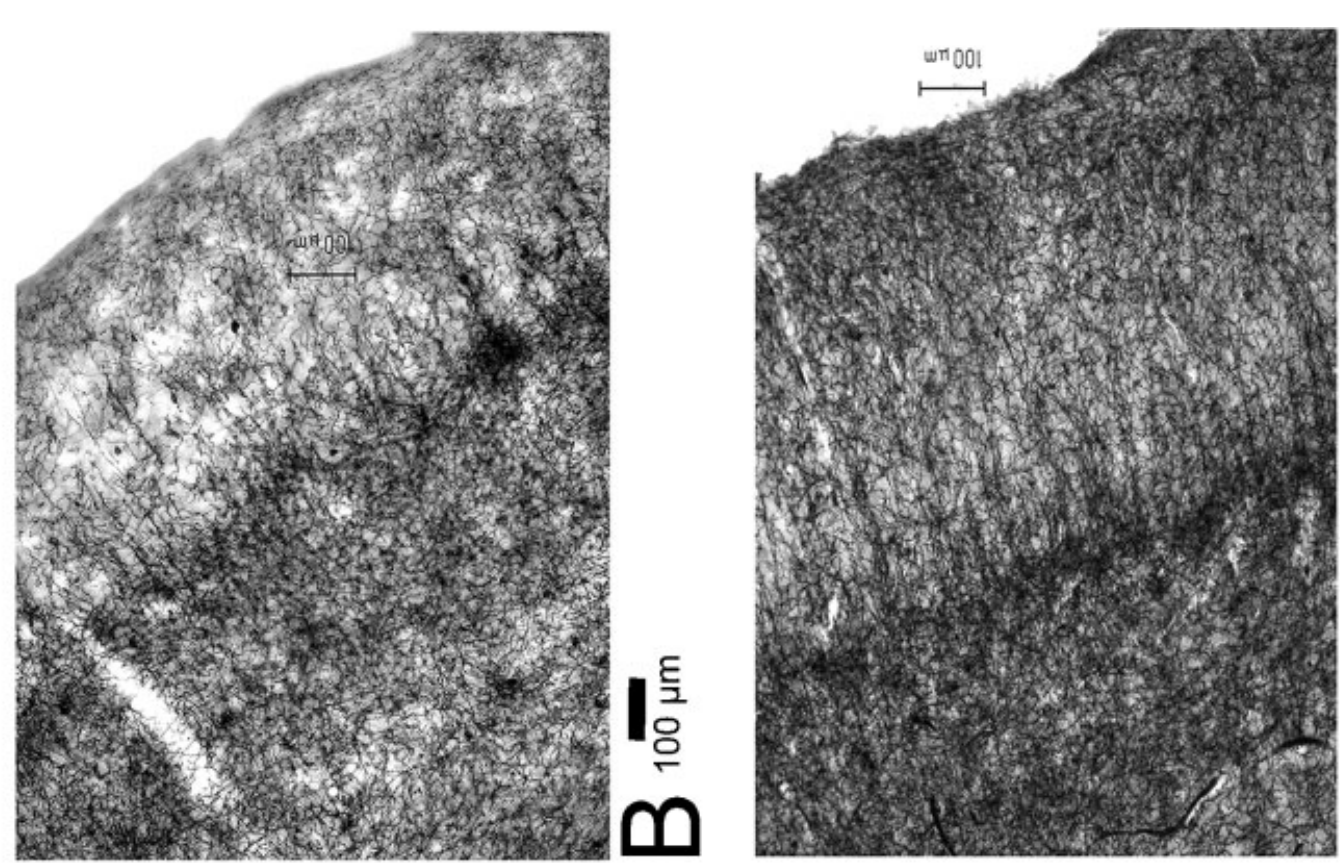
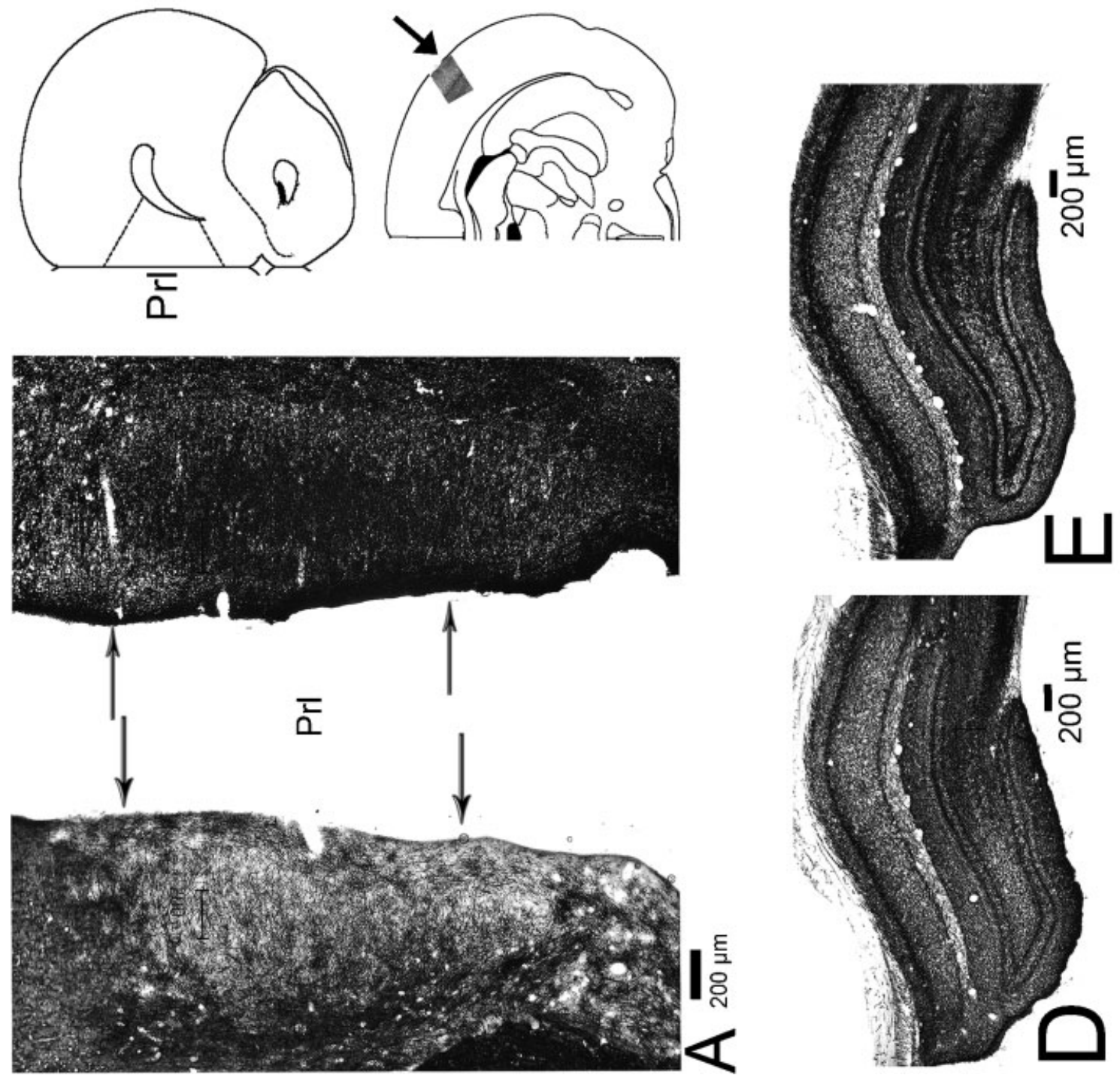
TABLE 2. Microsphere embolism-induced decreases in the density of AChE-positive axons two weeks after injection

\begin{tabular}{|c|c|c|c|c|c|c|c|c|c|}
\hline & \multicolumn{3}{|c|}{ Medial prefrontal cortex } & \multicolumn{3}{|c|}{ Frontoparietal cortex layers I-IV } & \multicolumn{3}{|c|}{ Frontoparietal cortex layers V-VI } \\
\hline & Ipsilateral & Contralateral & $\mathrm{I}-\mathrm{C} \Delta \%$ & Ipsilateral & Contralateral & $\mathrm{I}-\mathrm{C} \Delta \%$ & Ipsilateral & Contralateral & $\mathrm{I}-\mathrm{C} \Delta \%$ \\
\hline Sham & $28.8 \pm 1.1$ & $28.5 \pm 1.3$ & 1.1 & $23.3 \pm 1.2$ & $25.4 \pm 2.2$ & -8.3 & $23.4 \pm 1.3$ & $23.9 \pm 1.2$ & -2.1 \\
\hline $100 \mu \mathrm{L}$ & $16.1 \pm 1.1$ & $26.0 \pm 2.0$ & -38.1 & $15.2 \pm 1.4$ & $24.4 \pm 1.0$ & -37.7 & $16.9 \pm 2.1$ & $23.3 \pm 1.2$ & -27.5 \\
\hline Group $\Delta \%$ & $-44.1^{*}$ & -8.8 & & $-34.8^{*}$ & -3.9 & & $-27.8^{*}$ & -2.5 & \\
\hline $250 \mu \mathrm{L}$ & $13.8 \pm 1.2$ & $29.6 \pm 2.5$ & -53.3 & $13.9 \pm 0.6$ & $26.9 \pm 1.0$ & -48.3 & $16.4 \pm 1.4$ & $24.5 \pm 1.4$ & -33.1 \\
\hline Group $\Delta \%$ & $-52.1^{*, \dagger}$ & 3.9 & & $-40.3^{*}$ & 5.9 & & $-29.9^{*}$ & 2.5 & \\
\hline
\end{tabular}

Data are presented as mean numbers of axons $\pm \mathrm{SEM}$, and as percentage increases (Group $\Delta \%$ vs. sham; $\mathrm{I}-\mathrm{C} \Delta \%$ ipsilateral vs. contralateral). $* P<0.02$ compared with sham-lesioned controls; Kruskal-Wallis test; ${ }^{\dagger} P<0.05$ compared with the effects of $100 \mu \mathrm{L}$.

period, body weights did not differ between the two groups $(U=13.00, \quad P>0.05 ; \quad$ sham, $362.71 \pm 7.95 \mathrm{~g} ; \quad$ embolized, $348.57 \pm 4.60 \mathrm{~g})$.

\section{Presurgery performance}

Based on the data from the last five sessions prior to surgery, attentional performance did not differ between rats assigned for shamsurgery vs. microsphere embolism; hits, $F_{1,14}=0.71, P>0.05$; correct rejections, $F_{1,14}=0.05, P>0.05$; omissions, $F_{1,14}=0.15$, $P>0.05)$. Animals' ability to respond correctly in signal trials was signal duration-dependent $\left(F_{2,15}=91.02, P<0.001 ; 500 \mathrm{~ms}\right.$ signals, $66.92 \pm 2.63 \%$ hits; $50 \mathrm{~ms}$ signals, $44.52 \pm 2.37 \%$; $25 \mathrm{~ms}$ signals, $37.93 \pm 2.22 \%)$. Animals generated $73.17 \pm 2.43 \%$ correct rejections, and they omitted $13.83 \pm 1.42$ trials/session (or $8.53 \%$ of all trials).

The animals' hit rate varied over blocks of trials and in interaction with signal length (block, $F_{4,15}=35.40, P<0.001$; block $\times$ signal duration, $F_{4,15}=0.01, P<0.02$ ), indicating that the hit rate to $500 \mathrm{~ms}$ and $50 \mathrm{~ms}$ signals, but not to $25 \mathrm{~ms}$ signals, decreased across blocks of trials (posthoc ANOVAs on the effects of block per signal duration: $500 \mathrm{~ms}, \quad F_{2,15}=3.94, P<0.05 ; 50 \mathrm{~ms}, \quad F_{2,15}=6.96$, $\left.P<0.01 ; 25 \mathrm{~ms}, F_{2,15}=0.15, P>0.05\right)$.

\section{Postsurgery performance}

Sham-operated animals required a smaller number of sessions $(1.88 \pm 0.35$; Mean \pm SEM) of postoperative training than embolized animals $(5.38 \pm 1.21 ; U=12.00, P=0.03)$ to reach asymptotic postsurgery performance (as defined in Materials and methods).

Microsphere embolism impaired the animals' ability to respond correctly in signal trials $\left(F_{1,14}=9.94, \quad P<0.01\right.$; sham, $55.38 \pm 3.81 \%$ hits; embolized, $31.34 \pm 6.01 \%$ hits). The embolisminduced impairment in the animals' hit rate interacted with signal duration $\left(F_{2,14}=12.23, P<0.001\right)$, reflecting the greater embolisminduced decrease of hits to $500 \mathrm{~ms}$ when compared to shorter signals (Fig. 7). However, posthoc ANOVAs indicated that embolism significantly reduced the animals' hit rate to all signal durations $(500 \mathrm{~ms}$, $F_{1,14}=15.53, P<0.002 ; 50 \mathrm{~ms}, F_{1,14}=6.63, P<0.05 ; 25 \mathrm{~ms}$, $F_{1,14}=5.747, P<0.05$ ), while the hit rate remained signal durationdependent in both groups (embolized, $F_{2,14}=18.03, P<0.001$; sham, $\left.F_{2,14}=60.55, P<0.001\right)$.

Microsphere embolism did not affect the animals' ability to respond correctly to non-signal trials $\left(F_{1,14}=3.23, P>0.05\right.$; see correct rejections in Fig. 7). Likewise, the number of omissions remained similar to that of sham-operated animals $\left(F_{1,14}=0.06, P>0.05\right.$; embolized, $10.2 \pm 2.33$ omissions/session; sham, $13.6 \pm 3.6$ omissions/session).

Time-on-task, as indicated by the effects of block (three blocks of 54 trials each; see Materials and methods) differentially affected the ability of sham-operated and embolized animals to respond to signals (signal duration, $F_{2,14}=44.63, P<0.001$; embolism, $F_{1,14}=7.15, \quad P<0.05 ; \quad$ block $\times$ signal duration $\times$ embolism; $\left.F_{4,16}=3.78, \quad P<0.02\right)$. Posthoc ANOvAs indicated significant interactions between the effects of embolism and block for the relative number of hits to $25 \mathrm{~ms}$ only $\left(25 \mathrm{~ms}, F_{2,14}=5.01\right.$, $P<0.02 ; 500 \mathrm{~ms}, F_{2,14}=2.75, P>0.05 ; 50 \mathrm{~ms}, \quad F_{2,14}=1.34$, $P>0.05)$. Inspection of the data suggested that this interaction was due to a decrease in the relative number of hits to shortest signals from the first to the second block of trials in embolized rats, but not in control animals.

\section{Distractor effects}

The effects of a visual distractor on attentional performance were assessed in order to specify further the nature of the impairments in performance produced by microsphere embolism. As expected, presentation of the distractor decreased the hit rate in all animals $\left(F_{1,14}=20.55, P<0.001\right.$; standard test sessions, $43.36 \pm 4.63 \%$ hits; distractor sessions, $34.54 \pm 3.87 \%$ hits). However, when compared with the detrimental effects of the distractor in sham-operated controls, the performance of embolized animals remained relatively insensitive to the distractor, as indicated by a significant interaction between the effects of embolism, distractor, and signal length $\left(F_{2,8}=5.64\right.$, $P<0.01$; see Fig. 7).

Posthoc independent sample $t$-tests revealed that presentation of the distracter significantly reduced the hit rate to $500 \mathrm{~ms}\left(t_{7}=10.00\right.$, $P<0.001)$ and $50 \mathrm{~ms}$ signals $\left(t_{7}=5.72, P<0.002\right)$ in shamoperated animals only (Fig. 7). Presentation of the distractor also reduced the animals' ability to reject non-signal events $\left(F_{1,14}=12.87\right.$, $P<0.01$; Fig. 7), but this effect did not differ between embolized and sham-operated animals $\left(F_{1,14}=3.29, P>0.05\right)$. Likewise, omissions were not affected by the distractor $\left(F_{1,14}=0.140, P>0.05\right.$; group $\times$ distractor: $\left(F_{1,8}=0.72, P>0.05\right)$.

\section{Cortical AChE-fibre density in task-performing animals and correlations with performance}

Task-performing rats were killed 16 weeks after infusion of the microsphere suspension. As summarized in Table 3, microsphere embolism decreased the density of cholinergic fibers in the cortex ipsilateral to the injection by $25-43 \%$ when compared with counts taken from the ipsilateral hemisphere of sham-operated controls or the contralateral hemispheres of embolized animals. This decrease matched the effects of embolism on AChE-positive fibre density obtained from animals injected with the $250 \mu \mathrm{L}$ dose and killed 2 weeks after injection (Table 2). 

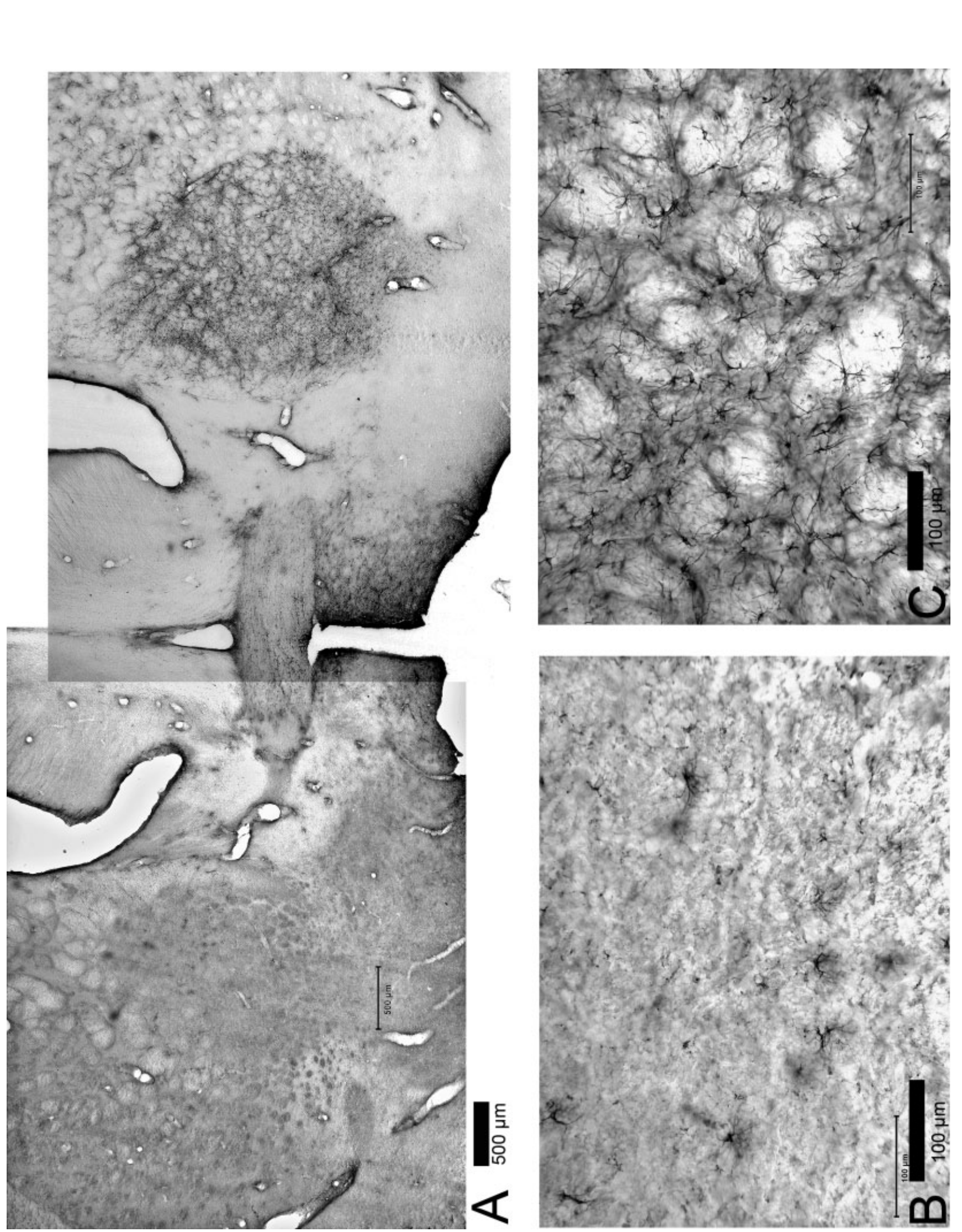

安

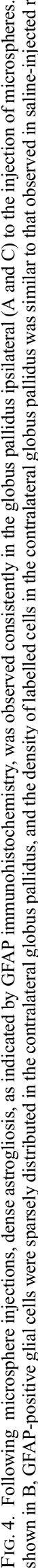



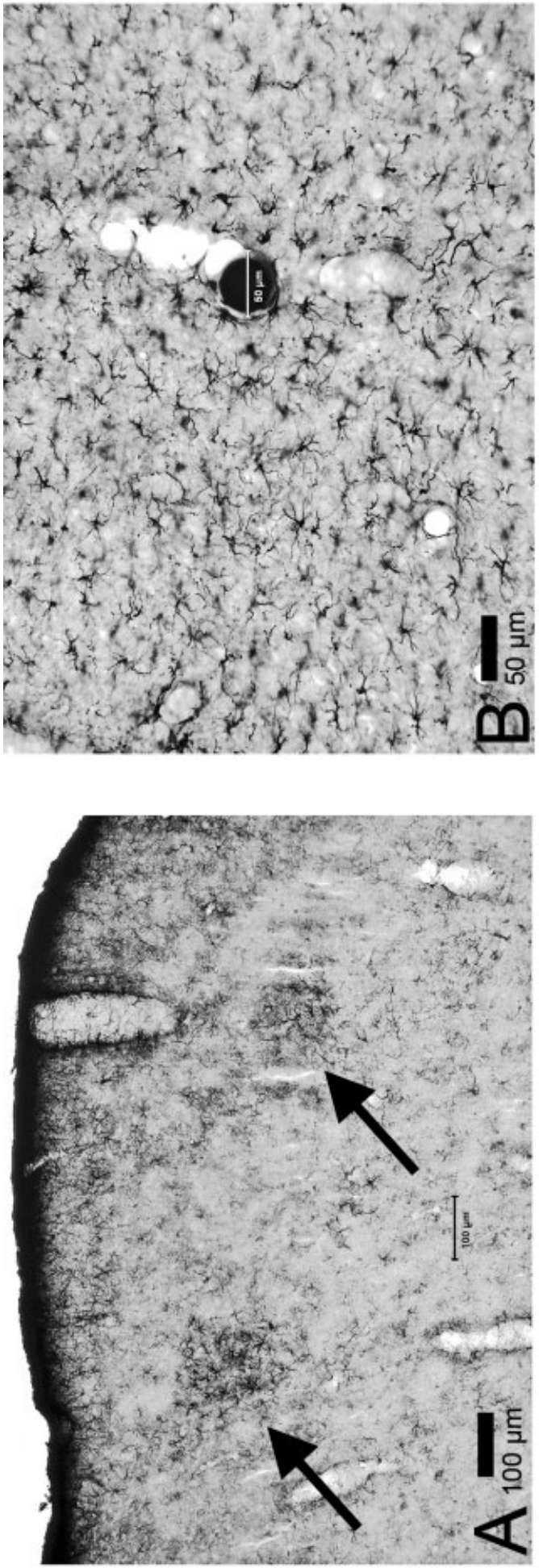
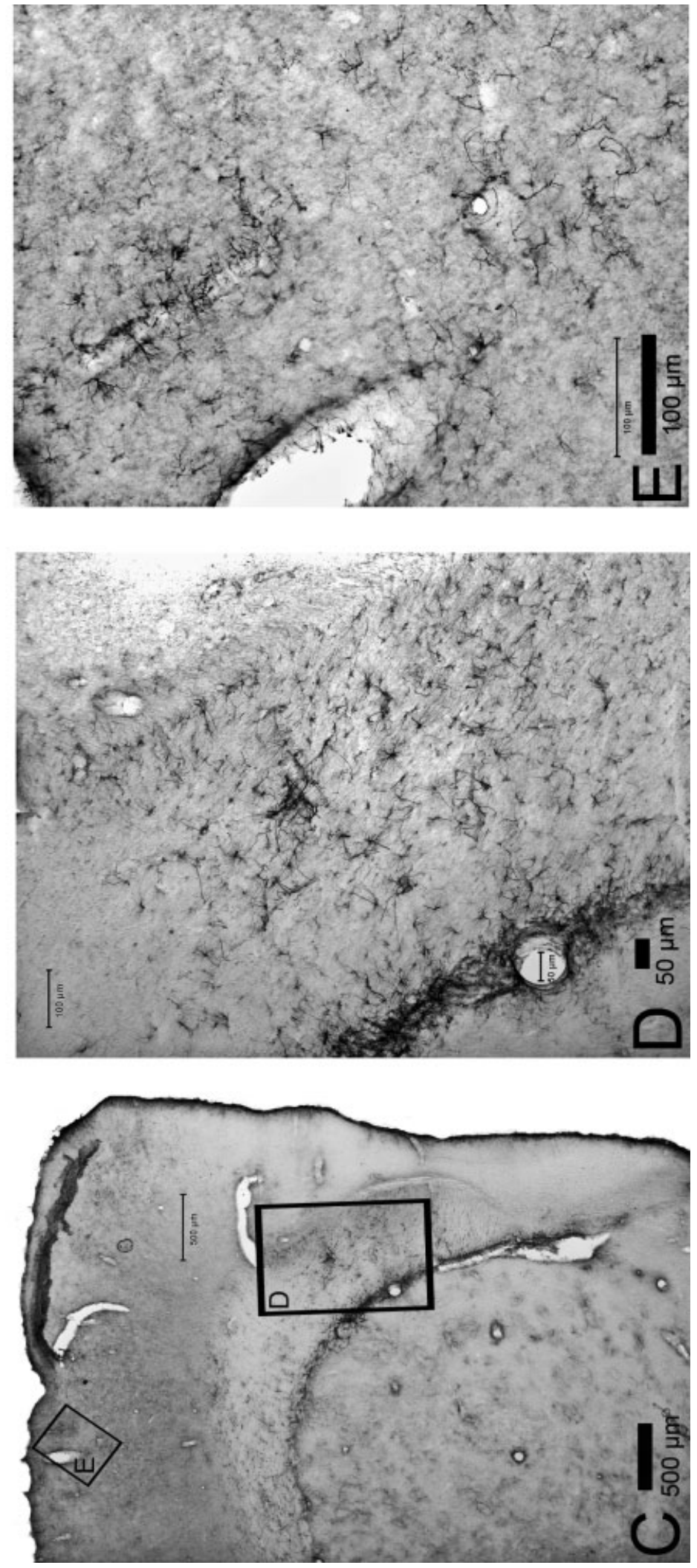

프. $. \Xi . \Xi$

क च ठ

唐

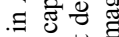

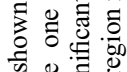

फ

突 $\cong$

풍 훙

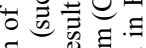

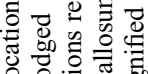

으으.융

Ð

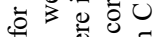

m os 0.

일 훙

$\exists$ के

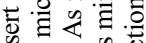

$\Xi \dot{0}$ टे ट

.气 월

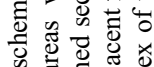

踪

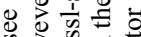

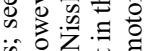

立出言

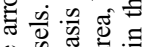

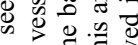

¿

음 뭉 뭉

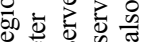

플 है

$\stackrel{2}{*} \div$

额

可总齐

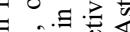

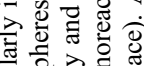

흥 층을

氖可

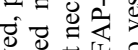

然苋䍐兑

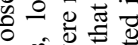

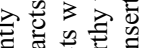

현 है

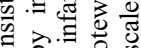

열 육

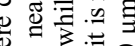

$\exists=0$

$\frac{0}{0} 00$

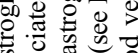

क

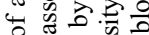

는?

류유.

造瑝

逮䒹专

8

Ð0ี

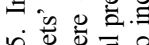

讨苍焉

至命苋 

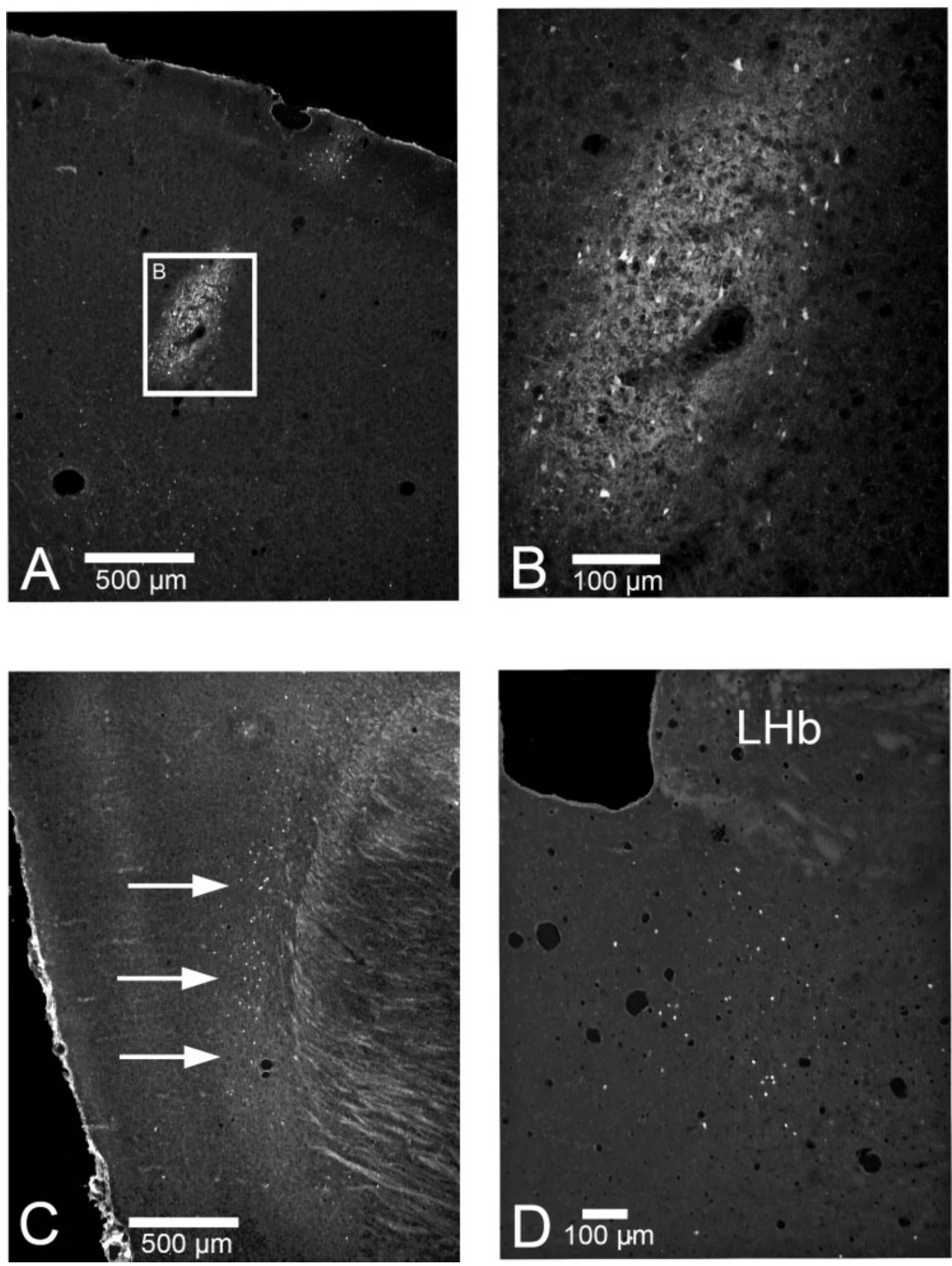

FIG. 6. Examination of Fluoro-Jade (FJ) stained sections confirmed the absence of massive areas of degeneration in the cortex and revealed, similar to the observations based on GFAP-stained sections (Fig. 5A), 'pockets' of degenerating neurons in the cortex (A represents frontoparietal cortex, magnified in B). Furthermore, and while GFAP-immunoreactivity did not indicate neurodegeneration in the medial prefrontal cortex, FJ-labelled neurons were observed on the deep layers of the prelimbic and anterior cingulate region (C; see the schematic insert in Fig. 3 illustrates the location of this area). With respect to subcortical regions, and outside the severely affected globus pallidus (see Fig. 4), FJ-labelled neurons were only sparsely observed, as exemplified in D that shows the mediodorsal thalamic nucleus ipsilateral to the microsphere injection ( $\mathrm{LHb}$, lateral habenular). 

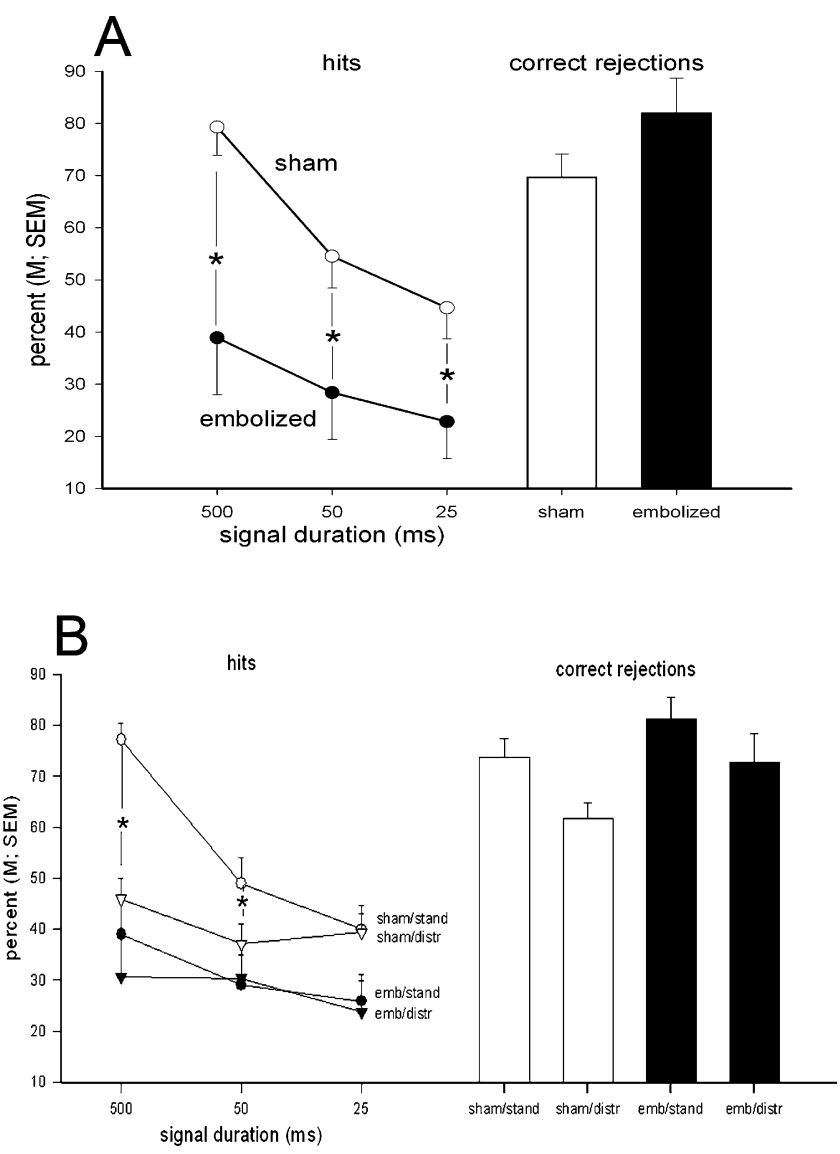

FIG. 7. (A) Effects of microsphere embolism on attentional performance (Mean \pm SEM). Embolized animals were impaired in their ability to detect signals (left panel). In contrast, the relative number of correct rejections was unaffected (right panel). (B) Effects of a distractor on the attentional performance of embolized and sham-operated control rats (Mean \pm SEM). Compared to the performance of sham-operated controls during standard sessions (sham/stand), presentation of the distractor impaired these animals' ability to detect $500 \mathrm{~ms}$ and $50 \mathrm{~ms}$ signals (sham/distr). In embolized animals $(\mathrm{emb})$, the distractor effect on hits was significantly attenuated. The distractor impaired all animals' ability to reject non-signal events (main effect of distractor; right panel). Stars $(\star)$ depict significant differences based on multiple comparisons that were conducted on the basis of significant results from ANOVAs (see Result section for statistical results).

The density of AChE-positive fibers in the ipsilateral medial prefrontal cortex of embolized, but not sham-operated, animals correlated significantly with the animals' ability to detect $500 \mathrm{~ms}$ and $50 \mathrm{~ms}$ signals (embolized: $500 \mathrm{~ms}, r=0.72, P<0.05 ; 50 \mathrm{~ms}$, $r=0.65, P<0.05$; sham: $500 \mathrm{~ms}, r=0.03 ; 50 \mathrm{~ms}, r=-0.23$; both $P \mathrm{~s}>0.05$; Fig. 8). No other significant correlations were found between measures of performance and AChE-positive fibre densities.

\section{Discussion}

Injections of microspheres into the common carotid artery resulted in a profound loss of cortical cholinergic inputs, particularly in prefrontal and frontoparietal regions. Furthermore, microsphere embolism impaired sustained attention performance, specifically by decreasing the animals' ability to respond correctly in signal trials. In embolized animals, the density of cortical cholinergic inputs correlated with attentional performance. As will be discussed below, the evidence based on GFAP immunohistochemistry and Fluoro-Jade histofluorescence suggests that the loss of cortical cholinergic inputs was probably a result of multiple, interacting pathological processes. Collectively, the present data suggest that the effects of microsphere embolism model central neuronal and cognitive aspects of vascular disease.

Microsphere embolism would be expected, and has been demonstrated, to affect multiple neurotransmitter and cellular systems and brain regions (references in Introduction; see also Takagi et al., 2002; Sekiguchi et al., 2003, 2005; Takeo et al., 2003; Date et al., 2004a,b). Thus, it should be reiterated that the present focus on establishing relationships between attentional performance and loss of cortical cholinergic inputs, and on utilizing additional histological evidence to inform hypotheses concerning the vulnerability of these neurons, is based on the intention to explore the usefulness of this animal model specifically for research on the interactions between microvascular disorder, cortical cholinergic inputs, and cognition (Sarter \& Bruno, 2004).

\section{Cortical cholinergic deafferentation}

The microsphere-induced cholinergic denervation of primarily medial and frontoparietal cortical regions may have been due to multiple and interacting pathological processes. The present evidence corresponds with the assumption that the majority of the microspheres moved through the internal carotid and the anterior and middle cerebral arteries and lodged primarily in the small vessels of medial frontal and parietal regions (Fig. 2), including the most dorsal cortical regions (Kataoka et al., 1991; Takagi et al., 1997a; Ward et al., 1998; Dihne et al., 2002). Indeed, occlusion of the anterior cerebral artery damaged the medial prefrontal cortex (Ward et al., 1998), and occlusion of the middle cerebral artery resulted in degeneration of primarily frontoparietal cortex (Ward et al., 1997). Although, as indicated on the basis of Nissl-, GFAP- and FJ-stained sections, microsphere embolism-induced loss of cholinergic projections to medial prefrontal and parietal regions was not associated with massive lesions such as those observed following artery occlusion, the restricted loss of cholinergic inputs to medial prefrontal and frontoparietal regions matches the cortical target regions primarily affected by artery occlusion.

Middle cerebral artery occlusion results in infarction and extensive degeneration of the GP (Kataoka et al., 1991; Dihne et al., 2002).

TABLE 3. Microsphere embolism-induced decreases in the density of AChE-positive axons in attentional task - performing animals 16 weeks after injection

\begin{tabular}{|c|c|c|c|c|c|c|c|c|c|}
\hline & \multicolumn{3}{|c|}{ Medial prefrontal cortex } & \multicolumn{3}{|c|}{ Frontoparietal cortex layers I-IV } & \multicolumn{3}{|c|}{ Frontoparietal cortex layers V-VI } \\
\hline & Ipsilateral & Contralateral & $\mathrm{I}-\mathrm{C} \Delta \%$ & Ipsilateral & Contralateral & $\mathrm{I}-\mathrm{C} \Delta \%$ & Ipsilateral & Contralateral & $\mathrm{I}-\mathrm{C} \Delta \%$ \\
\hline Sham & $26.6 \pm 1.0$ & $28.9 \pm 1.4$ & -2.7 & $18.2 \pm 0.2$ & $18.8 \pm 0.1$ & -3.2 & $19.9 \pm 1.8$ & $20.8 \pm 2.6$ & -4.3 \\
\hline $250 \mu \mathrm{L}$ & $15.0 \pm 1.0$ & $25.1 \pm 1.8$ & -40.3 & $11.9 \pm 2.0$ & $18.9 \pm 0.5$ & -37.0 & $14.9 \pm 0.5$ & $19.1 \pm 1.1$ & -26.1 \\
\hline Group $\Delta \%$ & $-43.6^{*}$ & -13.1 & & $-34.8^{*}$ & 0.5 & & $-25.1^{*}$ & -8.1 & \\
\hline
\end{tabular}

Data are presented as mean numbers of axons $\pm \mathrm{SEM}$, and as percentage increases (Group $\Delta \%$ vs. sham; I-C $\Delta \%$ ipsilateral vs. contralateral). ${ }^{*}$ Mann-Whitney $U$-test; $P<0.01$. 


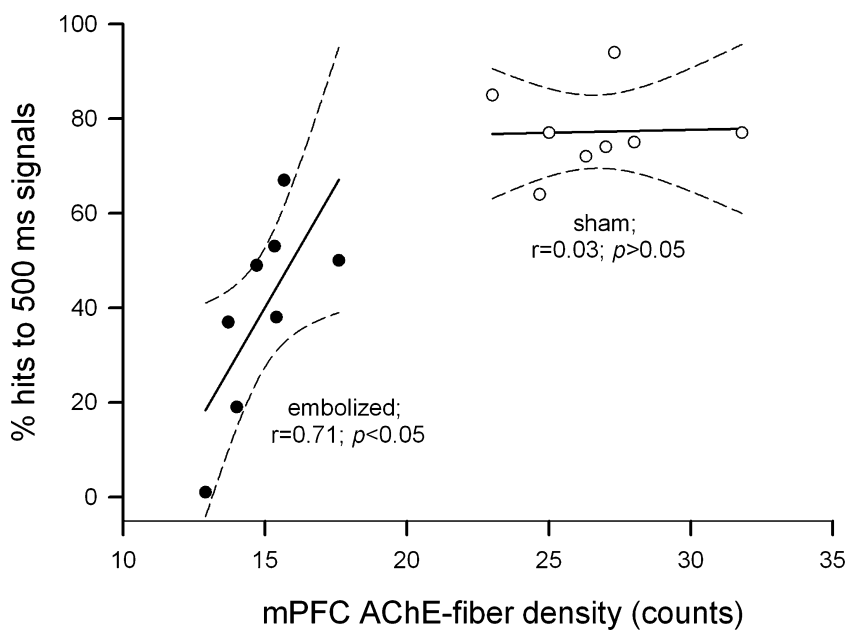

FIG. 8. Linear regression between AChE-positive fibre counts in the ipsilateral medial prefrontal cortex and the animals ability to detect longest $(500 \mathrm{~ms})$ signals (linear regression lines and 95\% confidence intervals are depicted for embolized and sham-operated rats; Pearson correlation coefficients are also indicated). In embolized rats, the detection rate to $500 \mathrm{~ms}$ and $50 \mathrm{~ms}$ (not shown) signals correlated significantly with residual fibre density in the medial prefrontal cortex.

However, infarcts in the GP were not consistently observed in the present experiment and thus are not considered a sufficient explanation for the astrogliosis seen in this region. Because of the absence of evidence indicating direct embolism-induced infarction of the GP, several pathological mechanisms may have interacted to yield this consistent finding. First, disruption of cerebral energy metabolism produced by microsphere embolism (Takeo et al., 1992; Miyake et al., 1993) may have caused retrograde degeneration of cortical projections from basal forebrain regions. Indeed, previous studies demonstrated that retrograde degeneration of cholinergic neurons results from neocortical infarction (Figueiredo et al., 1993; Liberini et al., 1994), or from distal occlusion of the middle cerebral artery (Barbelivien et al., 2004). While it is likely that non-cholinergic cortical projections arising from the basal forebrain also degenerated retrogradely, cholinergic neurons are exquisitely vulnerable to reduced metabolic supply in terminal regions, in part because of their relatively high energy consumption, particularly for mitochondrial acetyl-CoA synthesis (Milner et al., 1987; Takagi et al., 1997b; Szutowicz et al., 2000; Hartig et al., 2002). Second, GFAPand JD-stained sections indicated restricted infarcts and cell loss in cortical, specifically, frontoparietal regions (Figs 5 and 6). Although the underlying mechanisms are unclear, but may include disruption of trophic factor support (Ando et al., 2005), retrograde degeneration of cholinergic neurons has been demonstrated to either represent a direct consequence of cortical lesions, or cortical lesions render these neurons vulnerable to other pathological processes (Pearson et al., 1983; Sofroniew et al., 1990; Gutierrez et al., 1997). Third, the degree of infarction of the GP may have been underestimated by our histological analyses. Thus, collectively, the loss of cortical cholinergic inputs to medial prefrontal and frontoparietal regions (Tables 2 and 3) may have been a combined result of the vulnerability of these particular cortical target regions based on the primary passage of microspheres through anterior and middle cerebral arteries, disruption of cortical metabolic processes to which cholinergic neurons are particularly vulnerable and, in addition to these retrograde mechanisms, direct anterograde damage (see also Dihne et al., 2002).

\section{Impairments in attentional performance}

Microsphere embolism resulted in a decrease in the animals' ability to respond correctly in signal trials but did not affect their performance in non-signal trials. This pattern of impairment precisely mirrors the effects of immunotoxin-induced loss of cortical cholinergic inputs (McGaughy et al., 1996; McGaughy \& Sarter, 1998). Furthermore, the significant correlations between residual cortical cholinergic input density and the animals' hit rate substantiate the hypothesis that the attentional impairments produced by microsphere embolism were primarily due to cortical cholinergic deafferentation. The pattern of the behavioural effects does not suggest that sensorimotor neglect or lateralized response biases confounded the impairments in performance, because such biases would have also caused effects on the animals' performance in non-signal trials and would have attenuated the signal duration-dependent hit rate that remained present in embolized animals. The selectivity of the effects of microsphere embolism on the detection of signals conforms with hypotheses describing amplification of cortical sensory input processing by cholinergic inputs (Metherate \& Weinberger, 1990; Tremblay et al., 1990; Murphy \& Sillito, 1991; Hsieh et al., 2000; Penschuck et al., 2002; Oldford \& Castro-Alamancos, 2003), and the role of these inputs in attentional information processing in general (Everitt \& Robbins, 1997; Sarter et al., 2001, 2005).

The finding that such robust impairments in attentional performance were observed following right hemispheric embolism-induced cortical cholinergic deafferentation corresponds with recent evidence indicating that right-hemispheric, but not left-hemispheric, unilateral loss of cortical cholinergic inputs impaired the animals' ability to respond correctly in signal trials (Martinez \& Sarter, 2004). The present quantification of cortical cholinergic axons (Tables 2 and 3) did not indicate embolism-induced loss of cholinergic axons in the contralateral hemisphere, and thus the behavioural effects can be sufficiently explained on the basis of right cortical cholinergic deafferentation. Furthermore, as loss of predominately non-cholinergic neurons in the globus pallidus does not reproduce the effects of cholinergic lesions on attentional performance (Burk \& Sarter, 2001), the embolism-induced impairments in performance indeed may have been primarily due to the loss of basal forebrain cholinergic projections.

The finding that the distractor did not further impair the performance of embolized animals may have been due in part to a 'floor' effect (Fig. 7). Previous experiments demonstrated that even following an almost complete loss of cortical cholinergic inputs, the hit rate of lesioned animals remained at approximately $30 \%$, although, theoretically, zero percent hits are possible. Likewise, the effects of distractors on hits remained limited in animals with such lesions (McGaughy et al., 1996). The nature of this 'floor' effect is not understood, but it could reflect the contributions of other neuronal systems to the performance of signal trials. Alternatively, and based on evidence indicating that cortical cholinergic inputs to prefrontal regions mediate the processing of distractors (Gill et al., 2000), the microsphere-induced damage to this neuronal system may have contributed to the loss of distractor effects in embolized animals.

\section{Cortical cholinergic inputs, ischemia, and cognition}

The present data indicate the usefulness and significance of the effects of microsphere embolism as an animal model for investigating the reciprocal interactions between cholinergic neurotransmission and the cerebrovascular system, ischemia-induced neuronal damage, and cognitive functions. Microsphere embolism mimics the decline in cerebral perfusion during normal ageing and also the disruption of 
microvascular blood flow that represent critical steps in the development of dementia (Ballard et al., 2000; de la Torre \& Stefano, 2000; Farkas \& Luiten, 2001; Kalaria, 2002; Zekry et al., 2003; Langa et al., 2004).

As basal forebrain cholinergic projections innervate cortical microvessels (Bevan et al., 1982; Vaucher \& Hamel, 1995; Vaucher et al., 1997a) and contribute to the vasodilation of the cortex (Biesold et al., 1989; Lacombe et al., 1989; Linville et al., 1993; Vaucher \& Hamel, 1995; Vaucher et al., 1997a; Yamada et al., 2001), particularly in frontomedial and frontoparietal regions (Vaucher et al., 1997b; Barbelivien et al., 1999), a decline in the integrity of cortical cholinergic inputs would be expected to reduce cerebral blood flow (Waite et al., 1999), specifically the capacity of cortical blood flow to adapt to functional challenges (Linville \& Arneric, 1991; Tong \& Hamel, 1999), and thus to increase the vulnerability of the cortex to subsequent ischemic events (Farkas \& Luiten, 2001; Hartig et al., 2002). It should be mentioned that the absence of significant decreases in relative blood flow measured by Laser Doppler flowmetry, which is in contrast to the reduced flow observed after artery occlusion (e.g. Alkayed et al., 1998), may reflect the more subtle microvascular ischemia produced by microsphere embolism and/or an insufficient sensitivity of this technique to detect such ischemia (Miyake et al., 1993).

The present data suggest that ischemic events occurring in the region of the anterior and middle cerebral arteries primarily affect the integrity of cholinergic inputs in medial and parietal cortical regions. Thus, escalating reciprocal interactions between the decline in the functions and integrity of cortical cholinergic inputs, dysregulation of the cortical microvascular systems, amyloid precursor protein processing and $A \beta$ deposition (Zhang et al., 1997), and ischemic events, are hypothesized to mediate the rapid progression from early impairments in attentional functions to dementia (Sarter \& Bruno, 2004). The present data support the usefulness of microsphere embolism as an animal model to investigate these interactions.

\section{Acknowledgements}

This research was supported in part by PHS Grants NS37026, MH063114, KO2MH01072 (M.S.) and NS40267 (A.C.D.). Thanks to Terry Robinson (University of Michigan) for allowing us to use his microscopy equipment.

\section{Abbreviations}

ACh, acetylcholine; AChE, acetylcholinesterase; GFAP, glial fibrillary acidic protein; GP, globus pallidus; LDF, Laser Dopper flowmetry.

\section{References}

Alkayed, N.J., Harukuni, I., Kimes, A.S., London, E.D., Traystman, R.J. \& Hurn, P.D. (1998) Gender-linked brain injury in experimental stroke. Stroke, 29, 159-165.

Ando, T., Takagi, N., Takagi, K., Kago, T. \& Takeo, S. (2005) Effects of nefiracetam on the levels of brain-derived neurotrophic factor and synapsin I mRNA and protein in the hippocampus of microsphere-embolized rats. Eur. J. Pharmacol., 507, 49-56.

Apparsundaram, S., Martinez, V., Parikh, V., Kozak, R. \& Sarter, M. (2005) Increased capacity and density of choline transporters situated in synaptic membranes of the right medial prefrontal cortex of attentional taskperforming rats. J. Neurosci., 25, 3851-3856.

Arnold, H.M., Burk, J.A., Hodgson, E.M., Sarter, M. \& Bruno, J.P. (2002) Differential cortical acetylcholine release in rats performing a sustained attention task versus behavioral control tasks that do not explicitly tax attention. Neuroscience, 114, 451-460.
Ballard, C., McKeith, I., O’Brien, J., Kalaria, R., Jaros, E., Ince, P. \& Perry, R. (2000) Neuropathological substrates of dementia and depression in vascular dementia, with a particular focus on cases with small infarct volumes. Dement. Geriatr. Cogn. Disord., 11, 59-65.

Barbelivien, A., Bertrand, N., Besret, L., Beley, A., MacKenzie, E.T. \& Dauphin, F. (1999) Neurochemical stimulation of the rat substantia innominata increases cerebral blood flow (but not glucose use) through the parallel activation of cholinergic and non-cholinergic pathways. Brain Res., 840, 115-124.

Barbelivien, A., Vaussy, C., Marchalant, Y., Maubert, E., Bertrand, N., Beley, A., Roussel, S., Mackenzie, E.T. \& Dauphin, F. (2004) Degeneration of the basalocortical pathway from the cortex induces a functional increase in galaninergic markers in the nucleus basalis magnocellularis of the rat. J. Cereb. Blood Flow Metab., 24, 1255-1266.

Bevan, J.A., Buga, G.M., Florence, V.M., Gonsalves, A. \& Snowden, A. (1982) Distribution of choline acetyltransferase in cerebral and extracerebral cranial arteries of the cat. Its relationship to neurogenic atropine-sensitive dilation. Circ. Res., 50, 470-476.

Biesold, D., Inanami, O., Sato, A. \& Sato, Y. (1989) Stimulation of the nucleus basalis of Meynert increases cerebral cortical blood flow in rats. Neurosci. Lett., 98, 39-44.

Burk, J.A., Herzog, C.D., Porter, M.C. \& Sarter, M. (2002) Interactions between aging and cortical cholinergic deafferentation on attention. Neurobiol. Aging, 23, 467-477.

Burk, J.A. \& Sarter, M. (2001) Dissociation between the attentional functions mediated via basal forebrain cholinergic and GABAergic neurons. Neuroscience, 105, 899-909.

Bushnell, P.J., Chiba, A.A. \& Oshiro, W.M. (1998) Effects of unilateral removal of basal forebrain cholinergic neurons on cued target detection in rats. Behav. Brain Res., 90, 57-71.

Chu, Y., Cochran, E.J., Bennett, D.A., Mufson, E.J. \& Kordower, J.H. (2001) Down-regulation of trkA mRNA within nucleus basalis neurons in individuals with mild cognitive impairment and Alzheimer's disease. J. Comp. Neurol., 437, 296-307.

Date, I., Takagi, N., Takagi, K., Kago, T., Matsumoto, K., Nakamura, T. \& Takeo, S. (2004a) Hepatocyte growth factor attenuates cerebral ischemiainduced learning dysfunction. Biochem. Biophys. Res. Commun., 319, 11521158.

Date, I., Takagi, N., Takagi, K., Kago, T., Matsumoto, K., Nakamura, T. \& Takeo, S. (2004b) Hepatocyte growth factor improved learning and memory dysfunction of microsphere-embolized rats. J. Neurosci. Res., 78, 442-453

de la Torre, J.C. \& Stefano, G.B. (2000) Evidence that Alzheimer's disease is a microvascular disorder: the role of constitutive nitric oxide. Brain Res. Rev., 34, 119-136.

Dihne, M., Grommes, C., Lutzenburg, M., Witte, O.W. \& Block, F. (2002) Different mechanisms of secondary neuronal damage in thalamic nuclei after focal cerebral ischemia in rats. Stroke, 33, 3006-3011.

Everitt, B.J. \& Robbins, T.W. (1997) Central cholinergic systems and cognition. Annu. Rev. Psychol., 48, 649-684.

Fadel, J., Moore, H., Sarter, M. \& Bruno, J.P. (1996) Trans-synaptic stimulation of cortical acetylcholine release after partial 192 IgG-saporin-induced loss of cortical cholinergic afferents. J. Neurosci., 16, 6592-6600.

Farkas, E. \& Luiten, P.G. (2001) Cerebral microvascular pathology in aging and Alzheimer's disease. Prog. Neurobiol., 64, 575-611.

Figueiredo, B.C., Piccardo, P., Maysinger, D., Clarke, P.B. \& Cuello, A.C. (1993) Effects of acidic fibroblast growth factor on cholinergic neurons of nucleus basalis magnocellularis and in a spatial memory task following cortical devascularization. Neuroscience, 56, 955-963.

Fukatsu, T., Miyake-Takagi, K., Nagakura, A., Omino, K., Okuyama, N., Ando, T., Takagi, N., Furuya, Y. \& Takeo, S. (2002) Effects of nefiracetam on spatial memory function and acetylcholine and GABA metabolism in microsphere-embolized rats. Eur. J. Pharmacol., 453, 59-67.

Geula, C. (1998) Abnormalities of neural circuitry in Alzheimer's disease: hippocampus and cortical cholinergic innervation. Neurology, 51, S18S29.

Gharbawie, O.A. \& Whishaw, I.Q. (2003) Cholinergic and serotonergic neocortical projection lesions given singly or in combination cause only mild impairments on tests of skilled movement in rats: evaluation of a model of dementia. Brain Res., 970, 97-109.

Gill, T.M., Sarter, M. \& Givens, B. (2000) Sustained visual attention performance-associated prefrontal neuronal activity: evidence for cholinergic modulation. J. Neurosci., 20, 4745-4757.

Gutierrez, H., Miranda, M.I. \& Bermudez-Rattoni, F. (1997) Learning impairment and cholinergic deafferentation after cortical nerve growth factor deprivation. J. Neurosci., 17, 3796-3803. 
Haga, S., Haga, C., Aizawa, T. \& Ikeda, K. (2002) Neuronal degeneration and glial cell-responses following trimethyltin intoxication in the rat. Acta Neuropathol., 103, 575-582.

Harkany, T., Penke, B. \& Luiten, P.G. (2000) beta-Amyloid excitotoxicity in rat magnocellular nucleus basalis. Effect of cortical deafferentation on cerebral blood flow regulation and implications for Alzheimer's disease. Ann. N.Y. Acad. Sci., 903, 374-386.

Hartig, W., Bauer, A., Brauer, K., Grosche, J., Hortobagyi, T., Penke, B., Schliebs, R. \& Harkany, T. (2002) Functional recovery of cholinergic basal forebrain neurons under disease conditions: old problems, new solutions? Rev. Neurosci., 13, 95-165.

Holley, L.A., Turchi, J., Apple, C. \& Sarter, M. (1995) Dissociation between the attentional effects of infusions of a benzodiazepine receptor agonist and an inverse agonist into the basal forebrain. Psychopharmacology, 120, 99108.

Hopkins, K.J., Wang, G. \& Schmued, L.C. (2000) Temporal progression of kainic acid induced neuronal and myelin degeneration in the rat forebrain. Brain Res., 864, 69-80.

Hsieh, C.Y., Cruikshank, S.J. \& Metherate, R. (2000) Differential modulation of auditory thalamocortical and intracortical synaptic transmission by cholinergic agonist. Brain Res., 880, 51-64.

Kalaria, R. (2002) Similarities between Alzheimer's disease and vascular dementia. J. Neurol. Sci., 203-204, 29-34.

Kataoka, K., Hayakawa, T., Kuroda, R., Yuguchi, T. \& Yamada, K. (1991) Cholinergic deafferentation after focal cerebral infarct in rats. Stroke, 22, 1291-1296.

Knox, C.A. \& Oliveira, A. (1980) Brain aging in normotensive and hypertensive strains of rats. III. A quantitative study of cerebrovasculature. Acta Neuropathol., 52, 17-25.

Lacombe, P., Sercombe, R., Verrecchia, C., Philipson, V., MacKenzie, E.T. \& Seylaz, J. (1989) Cortical blood flow increases induced by stimulation of the substantia innominata in the unanesthetized rat. Brain Res., 491, 1-14.

Langa, K.M., Foster, N.L. \& Larson, E.B. (2004) Mixed dementia: emerging concepts and therapeutic implications. JAMA, 292, 2901-2908.

Lehericy, S., Hirsch, E.C., Cervera-Pierot, P., Hersh, L.B., Bakchine, S., Piette, F., Duyckaerts, C., Hauw, J.J., Javoy-Agid, F. \& Agid, Y. (1993) Heterogeneity and selectivity of the degeneration of cholinergic neurons in the basal forebrain of patients with Alzheimer's disease. J. Comp. Neurol., 330, 15-31.

Liberini, P., Pioro, E.P., Maysinger, D. \& Cuello, A.C. (1994) Neocortical infarction in subhuman primates leads to restricted morphological damage of the cholinergic neurons in the nucleus basalis of Meynert. Brain Res., 648 , $1-8$.

Linville, D.G. \& Arneric, S.P. (1991) Cortical cerebral blood flow governed by the basal forebrain: age-related impairments. Neurobiol. Aging, 12, 503-510.

Linville, D.G., Williams, S. \& Arneric, S.P. (1993) Basal forebrain control of cortical cerebral blood flow is independent of local cortical neurons. Brain Res., 622, 26-34.

Luiten, P.G., de Jong, G.I., Van der Zee, E.A. \& van Dijken, H. (1996) Ultrastructural localization of cholinergic muscarinic receptors in rat brain cortical capillaries. Brain Res., 720, 225-229.

Lysakowski, A., Wainer, B.H., Bruce, G. \& Hersh, L.B. (1989) An atlas of the regional and laminar distribution of choline acetyltransferase immunoreactivity in rat cerebral cortex. Neuroscience, 28, 291-336.

Martinez, V. \& Sarter, M. (2004) Lateralized attentional functions of cortical cholinergic inputs. Behav. Neurosci., 118, 984-991.

McGaughy, J., Kaiser, T. \& Sarter, M. (1996) Behavioral vigilance following infusions of 192 IgG-saporin into the basal forebrain: selectivity of the behavioral impairment and relation to cortical AChE-positive fiber density. Behav. Neurosci., 110, 247-265.

McGaughy, J. \& Sarter, M. (1995) Behavioral vigilance in rats: task validation and effects of age, amphetamine, and benzodiazepine receptor ligands. Psychopharmacology, 117, 340-357.

McGaughy, J. \& Sarter, M. (1998) Sustained attention performance in rats with intracortical infusions of 192 IgG-saporin-induced cortical cholinergic deafferentation: effects of physostigmine and FG 7142. Behav. Neurosci., 112, 1519-1525.

Mesulam, M. (2004) The cholinergic lesion of Alzheimer's disease: pivotal factor or side show? Learn. Mem., 11, 43-49.

Metherate, R. \& Weinberger, N.M. (1990) Cholinergic modulation of responses to single tones produces tone-specific receptive field alterations in cat auditory cortex. Synapse, 6, 133-145.

Meyer, J., Xu, G., Thornby, J., Chowdhury, M. \& Quach, M. (2002) Longitudinal analysis of abnormal domains comprising mild cognitive impairment (MCI) during aging. J. Neurol. Sci., 201, 19-25.
Milner, T.A., Aoki, C., Sheu, K.F., Blass, J.P. \& Pickel, V.M. (1987) Light microscopic immunocytochemical localization of pyruvate dehydrogenase complex in rat brain: topographical distribution and relation to cholinergic and catecholaminergic nuclei. J. Neurosci., 7, 3171-3190.

Miyake, K., Takeo, S. \& Kaijihara, H. (1993) Sustained decrease in brain regional blood flow after microsphere embolism in rats. Stroke, 24, 415-420.

Montaldi, D., Brooks, D.N., McColl, J.H., Wyper, D., Patterson, J., Barron, E. \& McCulloch, J. (1990) Measurements of regional cerebral blood flow and cognitive performance in Alzheimer's disease. J. Neurol. Neurosurg. Psychiary, 53, 33-38.

Mufson, E.J., Ma, S.Y., Cochran, E.J., Bennett, D.A., Beckett, L.A., Jaffar, S., Saragovi, H.U. \& Kordower, J.H. (2000) Loss of nucleus basalis neurons containing trkA immunoreactivity in individuals with mild cognitive impairment and early Alzheimer's disease. J. Comp. Neurol., 427, 19-30.

Mufson, E.J., Ma, S.Y., Dills, J., Cochran, E.J., Leurgans, S., Wuu, J., Bennett, D.A., Jaffar, S., Gilmor, M.L., Levey, A.I. \& Kordower, J.H. (2002) Loss of basal forebrain P75 (NTR) immunoreactivity in subjects with mild cognitive impairment and Alzheimer's disease. J. Comp. Neurol., 443, 136-153.

Murphy, P.C. \& Sillito, A.M. (1991) Cholinergic enhancement of direction selectivity in the visual cortex of the cat. Neuroscience, 40, 13-20.

Oldford, E. \& Castro-Alamancos, M.A. (2003) Input-specific effects of acetylcholine on sensory and intracortical evoked responses in the "barrel cortex' in vivo. Neuroscience, 117, 769-778.

Oshiro, W.M., Bushnell, P.J. \& Chiba, A.A. (2000) A comparison of the effects of bilateral and unilateral infusions of muscimol into the basal forebrain on cued detection of visual targets in rats. Behav. Neurosci., 114, 137-149.

Palmer, A.M. (1996) Neurochemical studies of Alzheimer's disease. Neurodegeneration, 5, 381-391.

Pappas, B.A., Bayley, P.J., Bui, B.K., Hansen, L.A. \& Thal, L.J. (2000) Choline acetyltransferase activity and cognitive domain scores of Alzheimer's patients. Neurobiol. Aging, 21, 11-17.

Pearson, R.C., Gatter, K.C. \& Powell, T.P. (1983) Retrograde cell degeneration in the basal nucleus in monkey and man. Brain Res., 261, 321-326.

Penschuck, S., Chen-Bee, C.H., Prakash, N. \& Frostig, R.D. (2002) In vivo modulation of a cortical functional sensory representation shortly after topical cholinergic agent application. J. Comp. Neurol., 452, 38-50.

Perry, E.K., Blessed, G., Tomlinson, B.E., Perry, R.H., Crow, T.J., Cross, A.J., Dockray, G.J., Dimaline, R. \& Arregui, A. (1981) Neurochemical activities in human temporal lobe related to aging and Alzheimer-type changes. Neurobiol. Aging, 2, 251-256.

Pinard, E., Nallet, H., MacKenzie, E.T., Seylaz, J. \& Roussel, S. (2002) Penumbral microcirculatory changes associated with peri-infarct depolarizations in the rat. Stroke, 33, 606-612.

Procter, A.W. (1996) Neurochemical correlates of dementia. Neurodegeneration, 5, 403-407.

Raszkiewicz, J.L., Linville, D.G., Kerwin, J.F. Jr, Wagenaar, F. \& Arneric, S.P. (1992) Nitric oxide synthase is critical in mediating basal forebrain regulation of cortical cerebral circulation. J. Neurosci. Res., 33, 129-135.

Reed, B.R. (2004) Vascular dementia. Arch. Neurol., 61, 433-435.

Sarter, M. \& Bruno, J.P. (2004) Developmental origins of the age-related decline in cortical cholinergic function and associated cognitive abilities. Neurobiol. Aging, 25, 1127-1139.

Sarter, M., Givens, B. \& Bruno, J.P. (2001) The cognitive neuroscience of sustained attention: where top-down meets bottom-up. Brain Res. Rev., 35, $146-160$.

Sarter, M., Hasselmo, M.E., Bruno, J.P. \& Givens, B. (2005) Unraveling the attentional functions of cortical cholinergic inputs: interactions between signal-driven and top-down cholinergic modulation of signal detection. Brain Res. Rev., 48, 98-111.

Schallert, T., Fleming, S.M., Leasure, J.L., Tillerson, J.L. \& Bland, S.T. (2000) CNS plasticity and assessment of forelimb sensorimotor outcome in unilateral rat models of stroke, cortical ablation, parkinsonism and spinal cord injury. Neuropharmacology, 39, 777-787.

Schmued, L.C. \& Hopkins, K.J. (2000) Fluoro-Jade B: a high affinity fluorescent marker for the localization of neuronal degeneration. Brain Res., 874, 123-130.

Sekiguchi, M., Sugiyama, Y., Takagi, K., Takagi, N., Takeo, S., Tanaka, O., Yamato, I., Torigoe, K. \& Nowakowski, R.S. (2003) Rapid appearance of pathological changes of neurons and glia cells in the cerebellum of microsphere-embolized rats. Brain Res., 978, 228-232.

Sekiguchi, M., Takagi, K., Takagi, N., Date, I., Takeo, S., Tanaka, O., Yamato, I., Kobashikawa, S., Torigoe, K. \& Nowakowski, R.S. (2005) Time course and sequence of pathological changes in the cerebellum of microsphereembolized rats. Exp. Neurol., 191, 266-275. 
Seshadri, S., Beiser, A., Selhub, J., Jacques, P.F., Rosenberg, I.H., D’Agostino, R.B., Wilson, P.W. \& Wolf, P.A. (2002) Plasma homocysteine as a risk factor for dementia and Alzheimer's disease. N. Engl. J. Med., 346, 476-483.

Sims, N.R., Bowen, D.M., Allen, S.J., Smith, C.C., Neary, D., Thomas, D.J. \& Davison, A.N. (1983) Presynaptic cholinergic dysfunction in patients with dementia. J. Neurochem., 40, 503-509.

Soblosky, J.S., Tabor, S.L., Matthews, M.A., Davidson, J.F., Chorney, D.A. \& Carey, M.E. (1996) Reference memory and allocentric spatial localization deficits after unilateral cortical brain injury in the rat. Behav. Brain Res., 80, 185-194.

Sofroniew, M.V., Galletly, N.P., Isacson, O. \& Svendsen, C.N. (1990) Survival of adult basal forebrain cholinergic neurons after loss of target neurons. Science, 247, 338-342.

Stichel, C.C. \& Singer, W. (1987) Quantitative analysis of the choline acetyltransferase-immunoreactive axonal network in the cat primary visual cortex. I. Adult cats. J. Comp. Neurol., 258, 91-98.

Szutowicz, A., Tomaszewicz, M., Jankowska, A., Madziar, B. \& Bielarczyk, H. (2000) Acetyl-CoA metabolism in cholinergic neurons and their susceptibility to neurotoxic inputs. Metab. Brain Dis., 15, 29-44.

Tago, H., Kimura, H. \& Maeda, T. (1986) Visualization of detailed acetylcholinesterase fiber and neuron staining in rat brain by a sensitive histochemical procedure. J. Histochem. Cytochem., 34, 1431-1438.

Takagi, N., Miyake, K., Taguchi, T., Sugita, N., Takagi, K., Tamada, H. \& Takeo, S. (1997b) Changes in cholinergic neurons and failure in learning function after microsphere embolism-induced cerebral ischemia. Brain Res. Bull., 43, 87-92.

Takagi, N., Miyake, K., Taguchi, T., Tamada, H., Takagi, K., Sugita, N. \& Takeo, S. (1997a) Failure in learning task and loss of cortical cholingergic fibers in microsphere-embolized rats. Exp. Brain Res., 114, 279-287.

Takagi, N., Miyake-Takagi, K., Takagi, K., Tamura, H. \& Takeo, S. (2002) Altered extracellular signal-regulated kinase signal transduction by the muscarinic acetylcholine and metabotropic glutamate receptors after cerebral ischemia. J. Biol. Chem., 277, 6382-6390.

Takagi, N., Tsuru, H., Yamamura, M. \& Takeo, S. (1995) Changes in striatal dopamine metabolism after microsphere embolism in rats. Stroke, 26, 11011106.

Takeo, S., Fukatsu, T., Miyake-Takagi, K., Takagi, N., Niimura, M., Nagakura, A., Ando, T. \& Tanonaka, K. (2003) Persistent effects of delayed treatment with nefiracetam on the water maze task in rats with sustained cerebral ischemia. J. Pharmacol. Exp. Ther, 304, 513-523.

Takeo, S., Taguchi, T., Tanonaka, K., Miyake, K., Horiguchi, T., Takagi, N. \& Fujimori, K. (1992) Sustained damage to energy metabolism of brain regions after microsphere embolism in rats. Stroke, 23, 62-68.

Terry, A.V. Jr \& Buccafusco, J.J. (2003) The cholinergic hypothesis of age and Alzheimer's disease-related cognitive deficits: recent challenges and their implications for novel drug development. J. Pharmacol. Exp. Ther, 306, 821-827.
Tong, X.K. \& Hamel, E. (1999) Regional cholinergic denervation of cortical microvessels and nitric oxide synthase-containing neurons in Alzheimer's disease. Neuroscience, 92, 163-175.

Tremblay, N., Warren, R.A. \& Dykes, R.W. (1990) Electrophysiological studies of acetylcholine and the role of the basal forebrain in the somatosensory cortex of the cat. II. Cortical neurons excited by somatic stimuli. J. Neurophysiol., 64, 1212-1222.

Turchi, J. \& Sarter, M. (2000) Cortical cholinergic inputs mediate processing capacity: effects of 192 IgG-saporin-induced lesions on olfactory span performance. Eur. J. Neurosci., 12, 4505-4514.

Turchi, J. \& Sarter, M. (2001) Antisense oligodeoxynucleotide-induced suppression of basal forebrain NMDA-NR1 subunits selectively impairs visual attentional performance in rats. Eur. J. Neurosci., 14, 103-117.

Vaucher, E., Borredon, J., Bonvento, G., Seylaz, J. \& Lacombe, P. (1997b) Autoradiographic evidence for flow-metabolism uncoupling during stimulation of the nucleus basalis of Meynert in the conscious rat. J. Cereb. Blood Flow Metab., 17, 686-694.

Vaucher, E. \& Hamel, E. (1995) Cholinergic basal forebrain neurons project to cortical microvessels in the rat: electron microscopic study with anterogradely transported Phaseolus vulgaris leucoagglutinin and choline acetyltransferase immunocytochemistry. J. Neurosci., 15, 7427-7441.

Vaucher, E., Linville, D. \& Hamel, E. (1997a) Cholinergic basal forebrain projections to nitric oxide synthase-containing neurons in the rat cerebral cortex. Neuroscience, 79, 827-836.

Waite, J.J., Holschneider, D.P. \& Scremin, O.U. (1999) Selective immunotoxininduced cholinergic deafferentation alters blood flow distribution in the cerebral cortex. Brain Res., 818, 1-11.

Ward, N.M., Sharkey, J. \& Brown, V.J. (1997) Assessment of sensorimotor neglect after occlusion of the middle cerebral artery in the rat. Behav. Neurosci., 111, 1133-1145.

Ward, N.M., Sharkey, J., Marston, H.M. \& Brown, V.J. (1998) Simple and choice reaction-time performance following occlusion of the anterior cerebral arteries in the rat. Exp. Brain Res., 123, 269-281.

Yamada, M., Lamping, K.G., Duttaroy, A., Zhang, W., Cui, Y., Bymaster, F.P., McKinzie, D.L., Felder, C.C., Deng, C.X., Faraci, F.M. \& Wess, J. (2001) Cholinergic dilation of cerebral blood vessels is abolished in M(5) muscarinic acetylcholine receptor knockout mice. Proc. Natl Acad. Sci. USA, 98, 14096-14101.

Zar, J.H. (1974) Biostatistical Analysis. Prentice Hall, Englewood Cliffs, N.J.

Zekry, D., Duyckaerts, C., Belmin, J., Geoffre, C., Herrmann, F., Moulias, R. \& Hauw, J.J. (2003) The vascular lesions in vascular and mixed dementia: the weight of functional neuroanatomy. Neurobiol. Aging, 24, 213-219.

Zhang, F., Eckman, C., Younkin, S., Hsiao, K.K. \& Iadecola, C. (1997) Increased susceptibility to ischemic brain damage in transgenic mice overexpressing the amyloid precursor protein. J. Neurosci., 17, 7655-7661.

Zlokovic, B.V. (2002) Vascular disorder in Alzheimer's disease: role in pathogenesis of dementia and therapeutic targets. Adv. Drug Deliv. Rev., 54, 1553-1559. 